\title{
Well-posedness of Initial Value Problem for Fourth Order Nonlinear Schrödinger Equation
}

\author{
Yu-Zhu Wang and Wenxu Ge
}

\begin{abstract}
In this paper, we study the well-posedness of initial value problem for fourth order nonlinear Schrödinger equation. By exploiting the Strichartz estimates, Kato's smoothing effect and the maximal function estimates for the linear Schrödinger operator, we establish the local and global well-posedness of initial value problem for fourth order nonlinear Schrödinger in homogeneous and nonhomogeneous Besov spaces. Moreover, the scattering result for small initial data is also obtained.
\end{abstract}

Keywords: Nonlinear Schrödinger equation, Initial value problem, Wellposedness, Besov spaces.

\section{INTRODUCTION}

It is well known that the nonlinear Schrödinger (NLS) equation models a wide range of physical phenomena including self-focusing of optical beams in nonlinear media, the modulation of monochromatic waves, propagation of Langmuir waves in plasmas, etc. The nonlinear Schrödinger equation plays an important role in many areas of applied physics, such as nonrelativistic quantum mechanics, laser beam propagation, Bose-Einstein condensates, and so on (see [23]).The initial value problem (IVP) or the initial-boundary value problem (IBVP) of the nonlinear Schrödinger equations on $\mathbb{R}^{n}$ have been extensively studied in the last two decades (e.g., see [3]-[6], [1], [8], [11], [13], [18], [20]-[22]). 
This paper concerns with the initial value problem for the fourth order nonlinear Schrödinger equation

$$
\begin{array}{cl}
i u_{t}+a \partial_{x}^{2} u+b \partial_{x}^{4} u= \pm \partial_{x} u^{k}, & x \in \mathbb{R}, t \in \mathbb{R} \\
u(x, 0)=u_{0}(x), & x \in \mathbb{R}
\end{array}
$$

where $a, b \in \mathbb{R}, k \in \mathbb{N}, u=u(x, t)$ is a complex valued functions of $(x, t), u_{0}(x)$ is the given complex value function.

(1.1) plays an important role in the nonlinear fiber optics [7]. Karpman [10] employed (1.1) describing the resonant radiation of solitons. This class of nonlinear Schrödinger equations has been widely applied in applied science such as deep water wave dynamics, plasma physics, optical communications and so on [2]. A large amount of interesting works has been devoted to the study of Cauchy problem to dispersive equations, such as [5-6, 8, 22] and references cited therein. Hao, Hsiao and Wang [5] studied the IVP for one-dimensional fourth order nonlinear Schrödinger equation

$$
i u_{t}=\partial_{x}^{4} u+P\left(\left(\partial_{x}^{\alpha} u\right)_{|\alpha| \leq 2},\left(\partial_{x}^{\alpha} \bar{u}\right)_{|\alpha| \leq 2}\right) .
$$

They investigated the local smoothing effects and established local wellposedness. Hao, Hsiao and Wang [6] again studied the local smoothing effects and well-posedness of the IVP for (1.3) in multi-dimensional spaces and obtained local well-posedness. Segata [22] investigated the IVP for fourth order nonlinear Schrödinger equation which describes the motion of the vortex filament

$$
i u_{t}+\partial_{x}^{2} u+\nu \partial_{x}^{4} u=F\left(u, \bar{u}, \partial_{x} u, \partial_{x} \bar{u}, \partial_{x}^{2} u, \partial_{x}^{2} \bar{u}\right)
$$

and established the IVP for (1.4) is locally well-posed in the space $H^{s}(\mathbb{R})\left(s \geq \frac{1}{2}\right)$ under the conditions $\nu<0$ and $\mu-\frac{\nu}{2}=0$. Here $F\left(u, \bar{u}, \partial_{x} u, \partial_{x} \bar{u}, \partial_{x}^{2} u, \partial_{x}^{2} \bar{u}\right)$ is given by

$$
\begin{aligned}
F= & -\frac{1}{2}|u|^{2} u-\frac{3 \mu}{4}|u|^{4} u+\left(-2 \mu+\frac{\nu}{2}\right)\left(\partial_{x} u\right)^{2} \bar{u} \\
& -(4 \mu+\nu)\left|\partial_{x} u\right|^{2} u+u^{2} \partial_{x}^{2} \bar{u}+(-2 \mu+\nu)\left|\partial_{x}^{2} u\right|^{2} \partial_{x}^{2} u .
\end{aligned}
$$

Huo and Jia [8] improved Segata's results.

In this paper, we will investigate the well-posedness on the IVP (1.1)-(1.2) in homogeneous and nonhomogeneous Besov spaces. More precisely speaking, we will prove that the IVP (1.1)- (1.2) has a unique solution in $C\left([-T, T] ; \dot{B}_{2, q}^{s_{k}}\right)$ and $C\left([-T, T] ; B_{2, q}^{s}\right)$ for some $T>0$. We will also show that the IVP (1.1)-(1.2) admits a unique global solution in the space $C\left(\mathbb{R} ; \dot{B}_{2, q}^{s_{k}}\right)$ and $C\left(\mathbb{R} ; B_{2, q}^{s}\right)$ provided certain 
norm of the initial data is suitably small. Here $s_{k}=\frac{1}{2}-\frac{3}{k-1}, s>s_{k}, k \geq 5$ and $1 \leq q \leq \infty$. By employing the method in the present paper initiated by Planchon in [19]-[21] and developed by Molinet and Ribaud in [16]-[17], and the Strichartz estimates, Kato's smoothing effect and the maximal function estimates for the linear Schrödinger operator, we establish well-posedness on the IVP (1.1)-(1.2) in homogeneous and nonhomogeneous Besov spaces. Noticing that $s_{k}$ is the critical value obtained from a similar scaling argument as that in [14]. Thus by similar considerations as in [14] we can expect that the lowest index $s_{k}$ for Besov spaces that we obtain is optimal, namely, if $s<s_{k}$ then it is reasonable to conjecture that well-posedness does not hold in either $\dot{B}_{2, q}^{s}(\mathbb{R})$ or $B_{2, q}^{s}(\mathbb{R})$ for any $1 \leq q \leq \infty$.

The paper is organized as follows. In Section 2, we state some notations and give some preliminaries. Section 3 is devoted to establishing the estimates for nonlinear terms. Section 4 is devoted to establishing the local and global well-posedness in homogeneous and nonhomogeneous Besov spaces. Finally, in Section 5 we obtain the scattering result for small initial data.

\section{Preliminaries}

In this section, we give some preliminaries.

2.1. Notations. Throughout this paper, we will use the following notations:

The Fourier transform of $f$ will be denoted by

$$
\hat{f}(\xi)=\int_{\mathbb{R}} e^{-i x \xi} f(x) d x .
$$

$L^{p}(1 \leq p \leq \infty)$ denotes the usual space of all $L^{p}(\mathbb{R})$-functions on $\mathbb{R}$ with norm $\|f\|_{L^{p}}$. $H^{s}$ denotes s order sobolev space on $\mathbb{R}$ with norm $\|f\|_{H^{s}}=\|(I-$ $\Delta)^{\frac{s}{2}} f\left\|_{L^{2}}=\right\|\left(1+|\xi|^{2}\right)^{\frac{s}{2}} \hat{f} \|_{L^{2}}$, where $s$ is a real number and $I$ is unitary operator. The Riesz potential of order $s$ is denoted by

$$
D_{x}^{s}=\left(|\xi|^{s} \hat{f}(\xi)\right)^{\vee} .
$$

We will use the space-time Lebesgue spaces $L_{x}^{p} L_{t}^{q}$ and $L_{t}^{q} L_{x}^{p}$ respectively equipped with the norms

$$
\|f\|_{L_{x}^{p} L_{t}^{q}}=\left(\int_{\mathbb{R}}\|f(x, \cdot)\|_{L^{q}}^{p} d x\right)^{\frac{1}{p}}
$$


and

$$
\|f\|_{L_{t}^{q} L_{x}^{p}}=\left(\int_{\mathbb{R}}\|f(\cdot, t)\|_{L^{p}}^{q} d t\right)^{\frac{1}{q}} .
$$

Sometimes we will need need the local in time versions of those spaces. We denote them by $L_{x}^{p} L_{T}^{q}$ and $L_{T}^{q} L_{x}^{p}$ and they are respectively equipped with the norms

$$
\begin{gathered}
\|f(x, t)\|_{L_{x}^{p} L_{T}^{q}}=\|\| f(x, \cdot)\left\|_{L^{q}([-T, T])}\right\|_{L^{p}(\mathbb{R})} \\
\|f(x, t)\|_{L_{T}^{q} L_{x}^{p}}=\|\| f(\cdot, t)\left\|_{L^{p}}\right\|_{L^{q}([-T, T])} .
\end{gathered}
$$

Now we recall the definition of the homogeneous and nonhomogeneous Besov spaces. Throughout this paper, let $\varphi \in \mathbb{S}(\mathbb{R})$ such that $\hat{\varphi}$ is supported by the set $\left\{\xi: \frac{1}{2} \leq|\xi| \leq 2\right\}$ and

$$
\sum_{j \in \mathbb{Z}} \hat{\varphi}\left(2^{-j} \xi\right)=1, \xi \neq 0
$$

Define $\psi$ by

$$
\hat{\psi}=1-\sum_{j \geq 1} \hat{\varphi}\left(2^{-j} \xi\right)
$$

and note that $\psi \in \mathcal{D}(\mathbb{R}), \hat{\psi}$ is supported by the ball $\{\xi|| \xi \mid \leq 2\}$ and $\hat{\psi}=1$ for $|\xi| \leq 1$. We denote now by $\Delta_{j}$ and $S_{j}$ the convolution operators whose symbols are respectively given by $\hat{\varphi}\left(2^{-j} \xi\right)$ and $\hat{\psi}\left(2^{-j} \xi\right)$. Also we define the operator $\tilde{\Delta}_{j}$ by

$$
\tilde{\Delta}_{j}=\Delta_{j-1}+\Delta_{j}+\Delta_{j+1}
$$

which satisfies

$$
\tilde{\Delta}_{j} \circ \Delta_{j}=\Delta_{j} .
$$

For $1 \leq p \leq \infty, 1 \leq q \leq \infty$, the homogeneous Besov space $\dot{B}_{p, q}^{s}(\mathbb{R})$ is defined by

$$
\dot{B}_{p, q}^{s}(\mathbb{R})=\left\{u \in \mathbb{S}^{\prime}(\mathbb{R}) \mid\left\|2^{j s}\right\| \Delta_{j} u\left\|_{L^{p}}\right\|_{l^{q}(\mathbb{Z})}<\infty\right\}
$$

and the nonhomogeneous Besov space $B_{p, q}^{s}(\mathbb{R})$ is defined by

$$
B_{p, q}^{s}(\mathbb{R})=\left\{u \in \mathbb{S}^{\prime}(\mathbb{R}) \mid\left\|S_{0} u\right\|_{L^{p}}+\left\|2^{j s}\right\| \Delta_{j} u\left\|_{L^{p}}\right\|_{l^{q}(\mathbb{N})}<\infty\right\} .
$$

It is well known that $\dot{B}_{2,2}^{s}(\mathbb{R})=\dot{H}^{s}(\mathbb{R})$ and $B_{2,2}^{s}(\mathbb{R})=H^{s}(\mathbb{R})$.

Also it is well known that for $-\frac{1}{2}<s<\frac{1}{2}$, the two following convergences hold

$$
f=\lim _{r \rightarrow+\infty} \sum_{j=-r}^{r} \Delta_{j}(f)=\sum_{j=-\infty}^{+\infty} \Delta_{j}(f)
$$


and

$$
S_{k}(f)=\lim _{r \rightarrow+\infty} \sum_{j=-r}^{k} \Delta_{j}(f)=\sum_{j=-\infty}^{k} \Delta_{j}(f) .
$$

Firstly, we consider the initial value problem for the linear schrödinger equation

$$
\begin{gathered}
i u_{t}+a \partial_{x}^{2} u+b \partial_{x}^{4} u=f(x, t), \\
u(x, 0)=u_{0} .
\end{gathered}
$$

(2.1)-(2.2) can be rewritten as

$$
u(x, t)=S(t) u_{0}-i \int_{0}^{t} S(t-\tau) f(x, \tau) d \tau .
$$

Here

$$
S(t) u_{0}=e^{i\left(a \partial_{x}^{2}+b \partial_{x}^{4}\right) t} u_{0}=\mathcal{F}_{\xi}^{-1}\left(e^{i\left(-a \xi^{2}+b \xi^{4}\right) t} \hat{u}_{0}\right) .
$$

2.2. Preliminaries. Lemma 2.1 Let $u_{0} \in \mathbb{S}(\mathbb{R}), a b \leq 0(b \neq 0)$, then we have

$$
\begin{aligned}
& \left\|D_{x}^{\frac{3}{2}} S(t) u_{0}\right\|_{L_{x}^{\infty} L_{t}^{2}} \leq C\left\|u_{0}\right\|_{L^{2}} . \\
& \left\|S(t) u_{0}\right\|_{L_{x}^{4} L_{t}^{\infty}} \leq C\left\|D_{x}^{\frac{1}{4}} u_{0}\right\|_{L^{2}} .
\end{aligned}
$$

Proof. (2.5) can be derived from Theorem 4.1 in [12], for which we omit the details. Making use of Theorem 2.5 in [12], we may obtain (2.6), here we omit the proof.

Lemma 2.2 Let $\mathrm{T}$ be a linear operator defined on space-time functions $g(x, t)$ by

$$
T g(t)=\int_{-\infty}^{+\infty} K(t, \tau) g(\tau) d \tau
$$

such that

$$
\|T g\|_{L_{x}^{p_{1}} L_{t}^{q_{1}}} \leq C\|g\|_{L_{x}^{p_{2}} L_{t}^{q_{2}}}
$$

Here

$$
\min \left(p_{1}, q_{1}\right)>\max \left(p_{2}, q_{2}\right) .
$$

Then

$$
\left\|\int_{0}^{t} K(t, \tau) g(\tau) d \tau\right\|_{L_{x}^{p_{1}} L_{t}^{q_{1}}} \leq C\|g\|_{L_{x}^{p_{2}} L_{t}^{q_{2}}} .
$$


For the proof of Lemma 2.2, see Lemma 2 in [17].

Lemma 2.3 Assume that $u_{0} \in \mathbb{S}(\mathbb{R}), a b \leq 0(b \neq 0), f \in \mathbb{S}\left(\mathbb{R}^{2}\right)$ and let $\left(p_{1}, q_{1}\right),\left(p_{2}, q_{2}\right) \in[4,+\infty) \times[2,+\infty]$ such that

$$
\frac{1}{p_{i}}+\frac{1}{2 q_{i}} \leq \frac{1}{4}
$$

then for all $j \in \mathbb{Z}$, we have

$$
2^{j\left(\frac{1}{p_{i}}+\frac{4}{q_{i}}-\frac{1}{2}\right)}\left\|S(t) \Delta_{j} u_{0}\right\|_{L_{x}^{p_{i}} L_{t}^{q_{i}}} \leq C\left\|\Delta_{j} u_{0}\right\|_{L^{2}}
$$

and

$$
2^{j\left(\frac{1}{p_{1}}+\frac{4}{q_{1}}-\frac{1}{2}\right)}\left\|\Delta_{j} \int_{0}^{t} S(t-\tau) \partial_{x} f(\tau) d \tau\right\|_{L_{x}^{p_{1}} L_{t}^{q_{1}}} \leq C 2^{j\left(\frac{3}{2}-\frac{1}{p_{2}}-\frac{4}{q_{2}}\right)}\left\|\Delta_{j} f\right\|_{L_{x}^{p_{2}^{\prime}} L_{t}^{q_{2}^{\prime}}} .
$$

Proof. From (2.5) and Bernstein inequality, we get

$$
\left\|S(t) \tilde{\Delta}_{j} u_{0}\right\|_{L_{x}^{\infty} L_{t}^{2}} \leq C 2^{-\frac{3}{2} j}\left\|\Delta_{j} u_{0}\right\|_{L^{2}} .
$$

Similarly, using Bernstein inequality and (2.6) yields

$$
\left\|S(t) \tilde{\Delta}_{j} u_{0}\right\|_{L_{x}^{4} L_{t}^{\infty}} \leq C 2^{\frac{1}{4} j}\left\|\Delta_{j} u_{0}\right\|_{L^{2}} .
$$

We consider the operator $T=S(t) \tilde{\Delta}_{j}$ and apply Riesz-Thorin theorem, for $\theta \in$ $[0,1]$, we obtain

$$
\left\|S(t) \tilde{\Delta}_{j} u_{0}\right\|_{L_{x}^{\frac{4}{1-\theta}} L_{t}^{\frac{2}{\theta}}} \leq C 2^{\frac{1-7 \theta}{4} j}\left\|\Delta_{j} u_{0}\right\|_{L^{2}} .
$$

Note that $\tilde{\Delta}_{j} \circ \Delta_{j}=\Delta_{j}$, from the above inequality we deduce

$$
\left\|S(t) \Delta_{j} u_{0}\right\|_{L_{x}^{\frac{4}{1-\theta}} L_{t}^{\frac{2}{\theta}}} \leq C 2^{\frac{1-7 \theta}{4} j}\left\|\Delta_{j} u_{0}\right\|_{L^{2}}
$$

It follows from Sobolev embedding theorem and Bernstein inequality that

$$
\begin{aligned}
\left\|S(t) \Delta_{j} u_{0}\right\|_{L_{x}^{p_{i}} L_{t}^{q_{i}}} & \leq C\left\|D_{x}^{\alpha} S(t) \Delta_{j} u_{0}\right\|_{L_{x}^{\frac{4}{1-\theta}}} L_{t}^{\frac{2}{\theta}} \\
& \leq C 2^{\frac{1-7 \theta}{4} j}\left\|D_{x}^{\alpha} \Delta_{j} u_{0}\right\|_{L^{2}} \\
& \leq C 2^{\frac{1-7 \theta}{4} j} 2^{\alpha j}\left\|\Delta_{j} u_{0}\right\|_{L^{2}},
\end{aligned}
$$

where

and where $\left(p_{i}, q_{i}\right)$ is given by

$$
0 \leq \alpha<\frac{1-\theta}{4}
$$

$$
\left\{\begin{array}{l}
\frac{1}{p_{i}}=\frac{1-\theta}{4}-\alpha \Leftrightarrow \alpha=\frac{1}{4}-\frac{1}{p_{i}}-\frac{1}{2 q_{i}} \\
\frac{1}{q_{i}}=\frac{\theta}{2} \Leftrightarrow \theta=\frac{2}{q_{i}} .
\end{array}\right.
$$


(2.9) follows from

$$
\frac{1-7 \theta}{4}+\alpha=\frac{1}{2}-\frac{1}{p_{i}}-\frac{4}{q_{i}}
$$

and $0 \leq \alpha<\frac{1-\theta}{4}$ with $0 \leq \theta \leq 1$ if and only if $\frac{1}{p_{i}}+\frac{1}{2 q_{i}} \leq \frac{1}{4}$ with $p_{i}<+\infty$. This has completed the proof of (2.9).

From (2.9) yields

$$
2^{j\left(\frac{1}{p_{i}}+\frac{4}{q_{i}}-\frac{1}{2}\right)}\left\|\partial_{x}^{\frac{1}{2}} S(t) \Delta_{j} u_{0}\right\|_{L_{x}^{p_{i}} L_{t}^{q_{i}}} \leq C 2^{j \frac{1}{2}}\left\|\Delta_{j} u_{0}\right\|_{L^{2}} .
$$

And by duality, we obtain

$$
\left\|\int_{-\infty}^{+\infty} \partial_{x}^{\frac{1}{2}} S(-\tau) \Delta_{j} f(\tau) d \tau\right\|_{L^{2}} \leq C 2^{j\left(\frac{1}{2}-\frac{1}{p_{i}}-\frac{4}{q_{i}}\right)} 2^{j \frac{1}{2}}\left\|\Delta_{j} f\right\|_{L_{x}^{p_{i}^{\prime}} L_{t}^{q_{i}^{\prime}}} .
$$

Choosing $g \in L_{x}^{p_{1}^{\prime}} L_{t}^{q_{1}^{\prime}}$ with $\|g\|_{L_{x}^{p_{1}^{\prime}} L_{t}^{q_{1}^{\prime}}} \leq 1$, using Hölder inequality and (2.15), we deduce

$$
\begin{aligned}
& \left|\left\langle\int_{-\infty}^{+\infty} \partial_{x} S(t-\tau) \Delta_{j} f(x, \tau) d \tau, g(x, t)\right\rangle\right| \\
& =\left|\int_{-\infty}^{+\infty}\left(\int_{\mathbb{R}} \partial_{x}^{\frac{1}{2}} S(-\tau) \Delta_{j} f(x, \tau) d \tau\right)\left(\int_{\mathbb{R}} \overline{\partial_{x}^{\frac{1}{2}} S(-t) \tilde{\Delta}_{j} g(x, t)} d t\right) d x\right| \\
& \leq\left\|\int_{\mathbb{R}} \partial_{x}^{\frac{1}{2}} S(-\tau) \Delta_{j} f(x, \tau) d \tau\right\|_{L^{2}}\left\|\int_{\mathbb{R}} \overline{\partial_{x}^{\frac{1}{2}} S(-t) \tilde{\Delta}_{j} g(x, t)} d t\right\|_{L^{2}} \\
& \leq C 2^{j\left(\frac{1}{2}-\frac{1}{p_{2}}-\frac{4}{q_{2}}\right)} 2^{j \frac{1}{2}}\left\|\Delta_{j} f\right\|_{L_{x}^{p_{2}^{\prime}} L_{t}^{q_{2}^{\prime}}} 2^{j\left(\frac{1}{2}-\frac{1}{p_{1}}-\frac{4}{q_{1}}\right)} 2^{j \frac{1}{2}}\|g\|_{L_{x}^{p_{1}^{\prime}} L_{t}^{q_{1}^{\prime}}} .
\end{aligned}
$$

It follows from the above inequality that

$$
2^{j\left(\frac{1}{p_{1}}+\frac{4}{q_{1}}-\frac{1}{2}\right)}\left\|\Delta_{j} \int_{-\infty}^{+\infty} S(t-\tau) \partial_{x} f(\tau) d \tau\right\|_{L_{x}^{p_{1}} L_{t}^{q_{1}}} \leq C 2^{j\left(\frac{3}{2}-\frac{1}{p_{2}}-\frac{4}{q_{2}}\right)}\left\|\Delta_{j} f\right\|_{L_{x}^{p_{2}^{\prime}} L_{t}^{q_{2}^{\prime}}} .
$$

Since (2.9) implies $\max \left(p_{2}^{\prime}, q_{2}^{\prime}\right)<2 \leq \min \left(p_{1}, q_{1}\right)$, using Lemma 2.2, we obtain (2.10). The Lemma is proved.

Lemma 2.4 Assume that $u_{0} \in \mathbb{S}(\mathbb{R}), a b \leq 0(b \neq 0), f \in \mathbb{S}\left(\mathbb{R}^{2}\right)$ and let $(p, q)$ satisfy

$$
\frac{1}{p}+\frac{1}{2 q} \leq \frac{1}{4}
$$

with $p<+\infty$. Then for all $j \in \mathbb{Z}$, we have

$$
\begin{gathered}
\left\|S(t) \Delta_{j} u_{0}\right\|_{L_{t}^{\infty} L_{x}^{2}} \leq C\left\|u_{0}\right\|_{L^{2}} . \\
\left\|\int_{0}^{t} \partial_{x} S(t-\tau) \Delta_{j} f(x, \tau) d \tau\right\|_{L_{t}^{\infty} L_{x}^{2}} \leq C 2^{j} 2^{j\left(\frac{1}{2}-\frac{1}{p}-\frac{4}{q}\right)}\left\|\Delta_{j} f\right\|_{L_{x}^{p^{\prime}} L_{t}^{q^{\prime}}} .
\end{gathered}
$$


Proof. Since the group $S(t)$ is unitary in $H^{s}(\mathbb{R})$, by Bernstein inequality, we know that (2.17) holds.

Using (2.9), we obtain

$$
2^{-j\left(\frac{1}{2}-\frac{1}{p}-\frac{4}{q}\right)}\left\|\partial_{x} S(t) \Delta_{j} u_{0}\right\|_{L_{x}^{p} L_{t}^{q}} \leq C 2^{j}\left\|\Delta_{j} u_{0}\right\|_{L^{2}}
$$

with $\frac{1}{p}+\frac{1}{2 q} \leq \frac{1}{4}$ and $p<+\infty$.

It follows from duality method that

$$
\left\|\int_{-\infty}^{+\infty} \partial_{x} S(-\tau) \Delta_{j} f(x, \tau) d \tau\right\|_{L^{2}} \leq C 2^{j} 2^{j\left(\frac{1}{2}-\frac{1}{p}-\frac{4}{q}\right)}\left\|\Delta_{j} f\right\|_{L_{x}^{p^{\prime}} L_{t}^{q^{\prime}}}
$$

Since $S(t)$ is unitary group in $L^{2}(\mathbb{R})$, which commutes with space derivatives, we get

$$
\sup _{t \in \mathbb{R}}\left\|\int_{-\infty}^{+\infty} \partial_{x} S(t-\tau) \Delta_{j} f(x, \tau) d \tau\right\|_{L^{2}} \leq C 2^{j} 2^{j\left(\frac{1}{2}-\frac{1}{p}-\frac{4}{q}\right)}\left\|\Delta_{j} f\right\|_{L_{x}^{p^{\prime}} L_{t}^{q^{\prime}}}
$$

For any fixed $t$, substituting $f(\tau)$ by the function $\chi_{R_{+}}(\tau) \chi_{\{\tau \leq t\}}(\tau) f(\tau)$ in the above inequality, we have completed the proof of (2.18).

Recall that we want to solve (1.1), (1.2) for initial data in the homogeneous Besov space $\dot{B}_{2, q}^{s_{k}}$ and in the nonhomogeneous Besov space $B_{2, q}^{s}$, where $s \geq s_{k}=$ $\frac{1}{2}-\frac{3}{k-1}, k \geq 5$ and $1 \leq q \leq \infty$.

Define function space

$$
E_{q}=\left\{u \in \mathbb{S}^{\prime}\left(\mathbb{R}^{2}\right) \mid\|u\|_{E_{q}}=N_{1, q}(u)+N_{2, q}(u)<+\infty\right\} .
$$

Here

$$
N_{i, q}(u)=\left\|2^{j s_{k}} 2^{j\left(\frac{1}{p_{i}}+\frac{4}{q_{i}}-\frac{1}{2}\right)}\right\| \Delta_{j} u\left\|_{L_{x}^{p_{i}} L_{t}^{q_{i}}}\right\|_{l^{q}}(\mathbb{Z}), \quad i=1,2
$$

and

$$
\left(p_{1}, q_{1}\right)=\left(\frac{4(k-1)}{3}, 2(k-1)\right), \quad\left(p_{2}, q_{2}\right)=(8,4)
$$

Sometime we also employ $E_{q, T}$ to denote the local in time version of $E_{q}$. Where $N_{i, q}(u)$ replaced respectively with

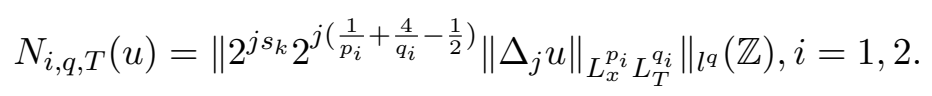

A direct computation, we obtain the following Lemma. 
Lemma 2.5 Let $\left(p_{i}, q_{i}\right), i=1,2$, be defined by $(2.22)$ and set $(p, q)=(8,4)$. Then the following conclusion hold

$$
\begin{gathered}
\frac{1}{p_{i}}+\frac{1}{2 q_{i}} \leq \frac{1}{4}, \quad p_{i}<+\infty \\
\frac{k-1}{p_{1}}+\frac{1}{p_{2}}=\frac{1}{p^{\prime}} . \\
\frac{k-1}{q_{1}}+\frac{1}{q_{2}}=\frac{1}{q^{\prime}} . \\
\frac{1}{2}-\frac{1}{p_{1}}-\frac{4}{q_{1}}>s_{k}
\end{gathered}
$$

and

$$
-\frac{1}{2}+\frac{1}{p_{2}}+\frac{4}{q_{2}}+(k-1)\left(s_{k}-\frac{1}{2}+\frac{1}{p_{1}}+\frac{4}{q_{1}}\right)>0 .
$$

Lemma 2.6 Assume that $u_{0} \in \dot{B}_{2, q}^{s_{k}}, q \in[1,+\infty]$ and $a b \leq 0(b \neq 0)$, then we have

$$
\left\|S(t) u_{0}\right\|_{L_{t}^{\infty} \dot{B}_{2, q}^{s_{k}}}+\left\|S(t) u_{0}\right\|_{E_{q}} \leq C\left\|u_{0}\right\|_{\dot{B}_{2, q}^{s_{k}}} .
$$

Proof. Using (2.17), we obtain

$$
\begin{aligned}
\left\|S(t) u_{0}\right\|_{L_{t}^{\infty} \dot{B}_{2, q}^{s_{k}}} & =\sup _{t \in \mathbb{R}}\left[\sum_{j \in \mathbb{Z}}\left(2^{j s_{k}}\left\|\Delta_{j} S(t) u_{0}\right\|_{L^{2}}\right)^{q}\right]^{\frac{1}{q}} \\
& \leq C\left[\sum_{j \in \mathbb{Z}}\left(2^{j s_{k}}\left\|\Delta_{j} u_{0}\right\|_{L^{2}}\right)^{q}\right]^{\frac{1}{q}} \\
& =C\left\|u_{0}\right\|_{\dot{B}_{2, q}^{s_{k}}} .
\end{aligned}
$$

Thanks to $(2.9)$, for $i=1,2$, we deduce

$$
\begin{aligned}
N_{i, q}\left(S(t) u_{0}\right) & =\left\|2^{j s_{k}} 2^{j\left(\frac{1}{p_{i}}+\frac{4}{q_{i}}-\frac{1}{2}\right)}\right\| \Delta_{j} S(t) u_{0}\left\|_{L_{x}^{p_{i}} L_{t}^{q_{i}}}\right\|_{l^{q}(\mathbb{Z})} \\
& \leq C\left\|2^{j s_{k}}\right\| \Delta_{j} u_{0}\left\|_{L^{2}}\right\|_{l^{q}(\mathbb{Z})} \\
& =C\left\|u_{0}\right\|_{\dot{B}_{2, q}^{s_{k}}}
\end{aligned}
$$

Combining (2.29), (2.30) and (2.20) yields (2.28). We have proved Lemma 2.6 . 


\section{Nonlinear estimates}

Lemma 3.1 Suppose that $u \in E_{q}, q \in[1,+\infty]$ and $a b \leq 0(b \neq 0)$. Then the following estimates hold

$$
\begin{gathered}
\left\|\int_{0}^{t} S(t-\tau) \partial_{x} u^{k}(\tau) d \tau\right\|_{L_{t}^{\infty} \dot{B}_{2, q}^{s_{k}}} \leq C\|u\|_{E_{q}}\|u\|_{E_{\infty}}^{k-1} . \\
\left\|\int_{0}^{t} S(t-\tau) \partial_{x} u^{k}(\tau) d \tau\right\|_{E_{q}} \leq C\|u\|_{E_{q}}\|u\|_{E_{\infty}}^{k-1} .
\end{gathered}
$$

Proof. Firstly, we establish the following estimate

$$
\left\|\Delta_{j}\left(u^{k}\right)\right\|_{L_{x}^{\frac{8}{7}} L_{t}^{\frac{4}{3}}} \leq C 2^{-j\left(s_{k}+\frac{3}{8}\right)} \gamma_{j}, \quad \forall j \in \mathbb{Z}
$$

where $\gamma_{j}(j \in \mathbb{R})$ satisfies

$$
\left\|\gamma_{j}\right\|_{l^{q}(\mathbb{Z})}=\left(\sum_{j \in \mathbb{Z}}\left|\gamma_{j}\right|^{q}\right)^{\frac{1}{q}} \leq\|u\|_{E_{q}}\|u\|_{E_{\infty}}^{k-1}
$$

For any fixed $t$, rewrite $\Delta_{j}\left(u^{k}(t)\right)$ as

$$
\begin{gathered}
\Delta_{j}\left(u^{k}(t)\right)=\Delta_{j}\left[\lim _{r \rightarrow+\infty}\left(S_{r}(u)\right)^{k}\right]=\Delta_{j}\left[\sum_{r \in \mathbb{Z}}\left(S_{r+1}(u)\right)^{k}-\left(S_{r}(u)\right)^{k}\right] \\
=\Delta_{j}\left\{\sum_{r \in \mathbb{Z}}\left[S_{r+1}(u)-S_{r}(u)\right] \sum_{p=0}^{k-1}\left(S_{r+1}(u)\right)^{p}\left(S_{r}(u)\right)^{k-p-1}\right\} \\
=\Delta_{j}\left[\sum_{r \in \mathbb{Z}} \Delta_{r+1} u \sum_{p=0}^{k-1}\left(S_{r+1}(u)\right)^{p}\left(S_{r}(u)\right)^{k-p-1}\right] .
\end{gathered}
$$

Since the term $\Delta_{r+1} u \sum_{p=0}^{k-1}\left(S_{r+1}(u)\right)^{p}\left(S_{r}(u)\right)^{k-p-1}$ is localzed in frequencies in a ball $|\xi| \leq 2^{k+r}$, we deduce that

$$
\Delta_{j}\left(u^{k}\right)=\Delta_{j}\left[\sum_{r=j-k}^{+\infty} \Delta_{r+1} u \sum_{p=0}^{k-1}\left(S_{r+1}(u)\right)^{p}\left(S_{r}(u)\right)^{k-p-1}\right]
$$


Noting that the operators $\Delta_{j}$ are uniformly bounded in $L^{p}(\mathbb{R})(p \in[1,+\infty])$, using Minkowski and Hölder inquality, we obtain

$$
\begin{aligned}
\left\|\Delta_{j}\left(u^{k}\right)\right\|_{L_{x}^{\frac{8}{7}} L_{t}^{\frac{4}{3}}} & \leq C\left\|\sum_{r=j-k}^{+\infty} \Delta_{r+1} u \sum_{p=0}^{k-1}\left(S_{r+1}(u)\right)^{p}\left(S_{r}(u)\right)^{k-p-1}\right\|_{L_{x}^{\frac{8}{7}} L_{t}^{\frac{4}{3}}}^{k-1}\left(S_{r+1}(u)\right)^{p}\left(S_{r}(u)\right)^{k-p-1} \|_{L_{x}^{\frac{8}{7}} L_{t}^{\frac{4}{3}}} \\
& \leq C \sum_{r=j-k}^{+\infty}\left\|\Delta_{r+1} u \sum_{p=0}^{+\infty}\right\| \Delta_{r+1} u\left\|_{L_{x}^{p_{2}} L_{t}^{q_{2}}} \sum_{p=0}^{k-1}\right\| S_{r+1}(u)\left\|_{L_{x}^{p_{1}} L_{t}^{q_{1}}}\right\| S_{r}(u) \|_{L_{x}^{p_{1}} L_{t}^{q_{1}}}^{k-p-1}, \\
& \leq C \sum_{r=j-k}^{+\infty},
\end{aligned}
$$

where we have used the following equality

$$
\begin{aligned}
& \frac{1}{p_{2}}+\frac{p}{p_{1}}+\frac{k-p-1}{p_{1}}=\frac{1}{p_{2}}+\frac{k-1}{p_{1}}=\frac{7}{8}, \\
& \frac{1}{q_{2}}+\frac{p}{q_{1}}+\frac{k-p-1}{q_{1}}=\frac{1}{q_{2}}+\frac{k-1}{q_{1}}=\frac{3}{4} .
\end{aligned}
$$

Let

$$
\alpha_{j}=2^{j s_{k}} 2^{j\left(\frac{1}{p_{2}}+\frac{4}{q_{2}}-\frac{1}{2}\right)}\left\|\Delta_{j} u\right\|_{L_{x}^{p_{2}} L_{t}^{q_{2}}}, \quad \tilde{\alpha}_{j}=2^{j s_{k}} 2^{j\left(\frac{1}{p_{1}}+\frac{4}{q_{1}}-\frac{1}{2}\right)}\left\|\Delta_{j} u\right\|_{L_{x}^{p_{1}} L_{t}^{q_{1}}}, j \in \mathbb{Z},
$$

then we have

$$
\left\|\Delta_{j} u\right\|_{L_{x}^{p_{2}} L_{t}^{q_{2}}}=2^{-j s_{k}} 2^{-j\left(\frac{1}{p_{2}}+\frac{4}{q_{2}}-\frac{1}{2}\right)} \alpha_{j}, j \in \mathbb{Z}
$$

and

$$
\left\|\Delta_{j} u\right\|_{L_{x}^{p_{1}} L_{t}^{q_{1}}}=2^{-j s_{k}} 2^{-j\left(\frac{1}{p_{1}}+\frac{4}{q_{1}}-\frac{1}{2}\right)} \tilde{\alpha}_{j}, j \in \mathbb{Z} .
$$

From $u \in E_{q}$, we get

$$
\|u\|_{N_{1, q}}=\left\|\tilde{\alpha}_{j}\right\|_{l^{q}(\mathbb{Z})},\|u\|_{N_{2, q}}=\left\|\alpha_{j}\right\|_{l^{q}(\mathbb{Z})},\|u\|_{N_{1, \infty}}=\left\|\tilde{\alpha}_{j}\right\|_{l^{\infty}(\mathbb{Z})} .
$$

Let

$$
\beta_{r}=\sum_{j=-\infty}^{r} 2^{(r-j)\left(s_{k}-\frac{1}{2}+\frac{1}{p_{1}}+\frac{4}{q_{1}}\right)} \tilde{\alpha}_{j}=\sum_{j=-\infty}^{+\infty} 2^{(r-j)\left(s_{k}-\frac{1}{2}+\frac{1}{p_{1}}+\frac{4}{q_{1}}\right)} \tilde{\alpha}_{j} \chi_{\{r-j \geq 0\}}
$$

with

$$
\chi_{\{r-j \geq 0\}}=1(r \geq j), \chi_{\{r-j \geq 0\}}=0(r<j) .
$$

Notice that $S_{r}(u)=\sum_{j=-\infty}^{r} \Delta_{j} u$, we obtain

$$
\left\|S_{r}(u)\right\|_{L_{x}^{p_{1} L_{t}^{q_{1}}}} \leq \sum_{j=-\infty}^{r}\left\|\Delta_{j} u\right\|_{L_{x}^{p_{1}} L_{t}^{q_{1}}}
$$




$$
=\sum_{j=-\infty}^{r} 2^{-j\left(s_{k}-\frac{1}{2}+\frac{1}{p_{1}}+\frac{4}{q_{1}}\right)} \tilde{\alpha}_{j}=2^{-r\left(s_{k}-\frac{1}{2}+\frac{1}{p_{1}}+\frac{4}{q_{1}}\right)} \beta_{r} .
$$

Since $s_{k}-\frac{1}{2}+\frac{1}{p_{1}}+\frac{4}{q_{1}}<0(k \geq 5)$, using Young inequality, we deduce

$$
\begin{gathered}
\left\|\beta_{r}\right\|_{l^{q}(\mathbb{Z})} \leq C\left\|\tilde{\alpha}_{j}\right\|_{l^{q}(\mathbb{Z})}=C\|u\|_{N_{1, q}}, \\
\left\|\beta_{r}\right\|_{l^{\infty}(\mathbb{Z})} \leq C\left\|\tilde{\alpha}_{j}\right\|_{l^{\infty}(\mathbb{Z})}=C\|u\|_{N_{1, \infty}} .
\end{gathered}
$$

Combining (3.7) and (3.9) yields

$$
\begin{aligned}
& \left\|\Delta_{r+1}(u)\right\|_{L_{x}^{p_{2}} L_{t}^{q_{2}}} \sum_{p=0}^{k-1}\left\|S_{r+1}(u)\right\|_{L_{x}^{p_{1}} L_{t}^{q_{1}}}^{p}\left\|S_{r}(u)\right\|_{L_{x}^{p_{1}} L_{t}^{q_{1}}}^{k-1} \\
\leq & 2^{-(r+1)\left(s_{k}-\frac{1}{2}+\frac{1}{p_{2}}+\frac{4}{q_{2}}\right)} \alpha_{r+1} \sum_{p=0}^{k-1} 2^{-p(r+1)\left(s_{k}-\frac{1}{2}+\frac{1}{p_{1}}+\frac{4}{q_{1}}\right)} \\
& \beta_{r+1}^{p} 2^{-(k-p-1) r\left(s_{k}-\frac{1}{2}+\frac{1}{p_{1}}+\frac{4}{q_{1}}\right)} \beta_{r}^{k-p-1} \\
= & 2^{-(r+1)\left(s_{k}-\frac{1}{2}+\frac{1}{p_{2}}+\frac{4}{q_{2}}\right)} 2^{-(k-1) r\left(s_{k}-\frac{1}{2}+\frac{1}{p_{1}}+\frac{4}{q_{1}}\right)} \\
& \alpha_{r+1} \sum_{p=0}^{k-1} 2^{-p\left(s_{k}-\frac{1}{2}+\frac{1}{p_{1}}+\frac{4}{q_{1}}\right)} \beta_{r+1}^{p} \beta_{r}^{k-p-1} \\
\leq & 2^{-(r+1)\left(s_{k}-\frac{1}{2}+\frac{1}{p_{2}}+\frac{4}{q_{2}}\right)} 2^{-(k-1)(r+1)\left(s_{k}-\frac{1}{2}+\frac{1}{p_{1}}+\frac{4}{q_{1}}\right)} \alpha_{r+1} \sum_{p=0}^{k-1} \beta_{r+1}^{p} \beta_{r}^{k-p-1} \\
\leq & C 2^{-(r+1)\left(s_{k}+\frac{3}{8}\right)} \alpha_{r+1}\left(\beta_{r+1}^{k-1}+\beta_{r}^{k-1}\right),
\end{aligned}
$$

In last inequality, we use

$$
\left(s_{k}-\frac{1}{2}+\frac{1}{p_{2}}+\frac{4}{q_{2}}\right)+(k-1)\left(s_{k}-\frac{1}{2}+\frac{1}{p_{1}}+\frac{4}{q_{1}}\right)=s_{k}+\frac{3}{8} .
$$

It follows from (3.6) and (3.12) that

$$
\begin{aligned}
\left\|\Delta_{j} u^{k}\right\|_{L_{x}^{\frac{8}{7}} L_{t}^{\frac{4}{3}}} & \leq C \sum_{r=j-k}^{+\infty}\left\|\Delta_{r+1} u\right\|_{L_{x}^{p_{2}} L_{t}^{q_{2}}} \sum_{p=0}^{k-1}\left\|S_{r+1}(u)\right\|_{L_{x}^{p_{2}} L_{t}^{q_{2}}}^{p}\left\|S_{r}(u)\right\|_{L_{x}^{p_{1}} L_{t}^{q_{1}}}^{k-p-1} \\
& \leq C \sum_{r=j-k}^{+\infty} 2^{-(r+1)\left(s_{k}+\frac{3}{8}\right)} \alpha_{r+1}\left(\beta_{r+1}^{k-1}+\beta_{r}^{k-1}\right) \\
& =C \sum_{r=j}^{+\infty} 2^{-(r-k+1)\left(s_{k}+\frac{3}{8}\right)} \alpha_{r-k+1}\left(\beta_{r-k+1}^{k-1}+\beta_{r-k}^{k-1}\right) \\
& =C \sum_{r=j}^{+\infty} 2^{-r\left(s_{k}+\frac{3}{8}\right)} \alpha_{r-k+1}\left(\beta_{r-k+1}^{k-1}+\beta_{r-k}^{k-1}\right) .
\end{aligned}
$$


Thus

$$
\begin{aligned}
& {\left[\sum_{j \in \mathbb{Z}}\left(2^{j\left(s_{k}+\frac{3}{8}\right)}\left\|\Delta_{j}\left(u^{k}\right)\right\|_{L_{x}^{\frac{8}{7}} L_{t}^{\frac{4}{3}}}\right)^{q}\right]^{\frac{1}{q}} } \\
\leq & C\left[\sum_{j \in \mathbb{Z}}\left(\sum_{r=j}^{+\infty} 2^{(j-r)\left(s_{k}+\frac{3}{8}\right)} \alpha_{r-k+1}\left(\beta_{r-k+1}^{k-1}+\beta_{r-k}^{k-1}\right)\right)^{q}\right]^{\frac{1}{q}} \\
\leq & C\left[\sum_{j \in \mathbb{Z}}\left(\sum_{r \in \mathbb{Z}}^{+\infty} 2^{j\left(s_{k}+\frac{3}{8}\right)} \chi_{\{j-r \leq 0\}} \alpha_{r-k+1}\left(\beta_{r-k+1}^{k-1}+\beta_{r-k}^{k-1}\right)\right)^{q}\right]^{\frac{1}{q}} .
\end{aligned}
$$

Since $s_{k}+\frac{3}{8}>0(k \geq 5)$, using Hölder inequality, (3.8) and (3.11) gives

$$
\begin{aligned}
{\left[\sum _ { j \in \mathbb { Z } } \left(2^{j\left(s_{k}+\frac{3}{8}\right)}\left\|\Delta_{j} u\right\|_{\left.\left.L_{x}^{\frac{8}{7}} L_{t}^{\frac{4}{3}}\right)^{q}\right]^{\frac{1}{q}}}\right.\right.} & \leq C\left[\sum_{r \in \mathbb{Z}} \alpha_{r-k+1}^{q}\left(\beta_{r-k+1}^{k-1}+\beta_{r-k}^{k-1}\right)^{q}\right]^{\frac{1}{q}} \\
& \leq C\|u\|_{N_{2, q}}\|u\|_{N_{1, \infty}}^{k-1} \leq C\|u\|_{E_{q}}\|u\|_{E_{\infty}}^{k-1} .
\end{aligned}
$$

This gives the proof of (3.3) and (3.4).

In what follows, we prove (3.1). For $p=8$ and $q=4, j \in \mathbb{Z}$, using (2.18) and (3.3), we obtain

$$
\begin{aligned}
& \left\|\Delta_{j} \int_{0}^{t} S(t-\tau) \partial_{x}\left(u^{k}(\tau)\right) d \tau\right\|_{L_{t}^{\infty} L_{x}^{2}}=\left\|\partial_{x} \int_{0}^{t} S(t-\tau) \Delta_{j}\left(u^{k}\right)(\tau) d \tau\right\|_{L_{t}^{\infty} L_{x}^{2}} \\
& \leq C 2^{j \frac{3}{8}}\left\|\Delta_{j}\left(u^{k}\right)\right\|_{L_{x}^{\frac{8}{7}} L_{t}^{\frac{4}{3}}} \leq C 2^{-j s_{k}} \gamma_{j} .
\end{aligned}
$$

From (3.13) and (3.4) yields

$$
\begin{aligned}
& \left\|\int_{0}^{t} S(t-\tau) \partial_{x}\left(u^{k}(t)\right) d \tau\right\|_{L_{t}^{\infty} \dot{B}_{2, q}^{s_{k}}} \\
= & \sup _{t \in \mathbb{R}}\left[\sum_{j \in \mathbb{Z}}\left(2^{j s_{k}}\left\|\Delta_{j} \int_{0}^{t} S(t-\tau) \partial_{x}\left(u^{k}(\tau)\right) d \tau\right\|_{L_{x}^{2}}\right)^{q}\right]^{\frac{1}{q}} \\
\leq & {\left[\sum_{j \in \mathbb{Z}}\left(2^{j s_{k}}\left\|\Delta_{j} \int_{0}^{t} S(t-\tau) \partial_{x}\left(u^{k}(\tau)\right) d \tau\right\|_{L_{t}^{\infty} L_{x}^{2}}\right)^{q}\right]^{\frac{1}{q}} } \\
\leq & C\left(\sum_{j \in \mathbb{Z}}\left|\gamma_{j}\right|^{q}\right)^{\frac{1}{q}} \\
\leq & C\|u\|_{E_{q}}\|u\|_{E_{\infty}}^{k-1} .
\end{aligned}
$$


Using (2.10) and (3.3), for $i=1,2$ and $j \in \mathbb{Z}$, we get

$$
\begin{aligned}
& 2^{j\left(\frac{1}{p_{i}}+\frac{4}{q_{i}}-\frac{1}{2}\right)}\left\|\Delta_{j} \int_{0}^{t} S(t-\tau) \partial_{x} u^{k}(\tau) d \tau\right\|_{L_{x}^{p_{i}} L_{t}^{q_{i}}} \\
\leq & C 2^{j\left(\frac{3}{2}-\frac{1}{p_{2}}-\frac{4}{q_{2}}\right)}\left\|\Delta_{j} u^{k}\right\|_{L_{x}^{p_{2}} L_{t}^{q_{2}^{\prime}}} \\
= & C 2^{j \frac{3}{8}}\left\|\Delta_{j} u^{k}\right\|_{L_{x}^{\frac{8}{7}} L_{t}^{\frac{4}{3}}} \\
\leq & C 2^{-j s_{k}} \gamma_{j} .
\end{aligned}
$$

By (2.24), (2.25), (3.4) and (3.14), we obtain

$$
\begin{aligned}
& \left\|\int_{0}^{t} S(t-\tau) \partial_{x} u^{k}(\tau) d \tau\right\|_{E_{q}} \\
= & \sum_{i=1}^{2}\left\|\int_{0}^{t} S(t-\tau) \partial_{x} u^{k}(\tau) d \tau\right\|_{N_{i, q}} \\
= & \sum_{i=1}^{2}\left[\sum_{j \in \mathbb{Z}}\left(2^{j s_{k}} 2^{j\left(\frac{1}{p_{i}}+\frac{4}{q_{i}}-\frac{1}{2}\right)}\left\|\Delta_{j} \int_{0}^{t} S(t-\tau) \partial_{x} u^{k}(\tau) d \tau\right\|_{\left.L_{x}^{p_{i}} L_{t}^{q_{i}}\right)^{q}}\right]^{\frac{1}{q}}\right. \\
\leq & C\left(\sum_{j \in \mathbb{Z}}\left|\gamma_{j}\right|^{q}\right)^{\frac{1}{q}} \leq C\|u\|_{E_{q}}\|u\|_{E_{\infty}}^{k-1} .
\end{aligned}
$$

This proves Lemma 3.1 .

Lemma 3.2 Assume that $u_{1}, u_{2} \in E_{q}$ and $a b \leq 0(b \neq 0)$, where $1 \leq q \leq \infty$. Then the following estimates hold

$$
\begin{aligned}
& \left\|\int_{0}^{t} S(t-\tau) \partial_{x}\left(u_{1}^{k}-u_{2}^{k}\right)(\tau) d \tau\right\|_{L_{t}^{\infty} \dot{B}_{2, q}^{s_{k}}} \leq C\left\|u_{1}-u_{2}\right\|_{E_{q}}\left(\left\|u_{1}\right\|_{E_{\infty}}^{k-1}+\left\|u_{2}\right\|_{E_{\infty}}^{k-1}\right) \\
& \left\|\int_{0}^{t} S(t-\tau) \partial_{x}\left(u_{1}^{k}-u_{2}^{k}\right)(\tau) d \tau\right\|_{E_{q}} \leq C\left\|u_{1}-u_{2}\right\|_{E_{q}}\left(\left\|u_{1}\right\|_{E_{\infty}}^{k-1}+\left\|u_{2}\right\|_{E_{\infty}}^{k-1}\right)
\end{aligned}
$$

Proof. From (3.5), we obtain

$$
\begin{gathered}
\Delta_{j}\left(u_{1}^{k}-u_{2}^{k}\right)=\Delta_{j}\left[\sum_{r=j-k}^{\infty} \Delta_{r+1}\left(u_{1}-u_{2}\right) \sum_{p=0}^{k-1}\left(S_{r+1}\left(u_{1}\right)\right)^{p}\left(S_{r}\left(u_{2}\right)\right)^{k-1-p}+\right. \\
\sum_{r=j-k}^{\infty} \Delta_{r+1}\left(u_{2}\right) S_{r+1}\left(u_{1}-u_{2}\right) \sum_{r_{1}+r_{2}+r_{3}=k-2}\left(S_{r+1}\left(u_{1}\right)\right)^{r_{1}}+\left(S_{r+1}\left(u_{2}\right)\right)^{r_{2}}\left(S_{r}\left(u_{1}\right)\right)^{r_{3}} \\
\left.+\sum_{r=j-k}^{\infty} \Delta_{r+1}\left(u_{2}\right) S_{r}\left(u_{1}-u_{2}\right) \sum_{r_{1}+r_{2}+r_{3}=k-2}\left(S_{r+1\left(u_{2}\right)}\right)^{r_{1}}\left(S_{r}\left(u_{1}\right)\right)^{r_{2}}\left(S_{r}\left(u_{2}\right)\right)^{r_{3}}\right]
\end{gathered}
$$

Similar to the proof of Lemma 3.1, we know that (3.15) and (3.16) hold. The Lemma is proved. 


\section{MAin ReSUlts}

In order to prove our main results, in what follows, we introduce four Lemmas.

Lemma 4.1 Assume that $a b \leq 0(b \neq 0), q \in[1,+\infty], m \in[4,+\infty]$ and $n \in$ $[2,+\infty]$ with $\frac{1}{m}+\frac{1}{2 n} \leq \frac{1}{4}$. Let $u$ be a solution of (1.1)- (1.2) with $\|u\|_{E_{q, T}}<\infty$. Then the sequence

$$
\alpha_{j}=2^{j s_{k}} 2^{j\left(\frac{1}{m}+\frac{4}{n}-\frac{1}{2}\right)}\left\|\Delta_{j} u\right\|_{L_{x}^{m} L_{t}^{n}}
$$

belongs to $l^{q}(\mathbb{Z})$ and the estimate holds

$$
\left\|\alpha_{j}\right\|_{l^{q}(\mathbb{Z})} \leq C\left(\left\|u_{0}\right\|_{\dot{B}_{2, q}^{s_{k}}}+\|u\|_{E_{q, T}}^{k}\right) .
$$

Proof. Since $u$ is a solution of (1.1)- (1.2), we have

$$
\Delta_{j} u=\Delta_{j}\left(S(t) u_{0}\right) \pm \Delta_{j}\left(\int_{0}^{t} S(t-\tau) \partial_{x} u^{k}(\tau) d \tau\right), \forall j \in \mathbb{Z}
$$

By (2.9), (3.2) and $E_{q, T} \hookrightarrow E_{\infty, T}$, we know that (4.2) holds. This proves Lemma 4.1 .

Lemma 4.2 Assume that $a b \leq 0(b \neq 0)$. Let $u$ be a solution of the problem (1.1)-(1.2), then we have

$$
\begin{aligned}
& \|u\|_{L_{T x}^{r}} \leq C(r) T^{\nu}\left(\left\|u_{0}\right\|_{\dot{B}_{2, q}^{s_{k}}}+\|u\|_{E_{q, T}}^{k}\right), \\
& r \in\left(\frac{k-1}{3}, \frac{5}{3}(k-1)\right) \bigcap\left(\frac{7(k-1)}{2 k-5},+\infty\right) \bigcap(4,+\infty) .
\end{aligned}
$$

Here $\nu=\nu(r)>0$.

Proof. It suffices to show that

$$
\sum_{j=-\infty}^{0}\left\|\Delta_{j} u\right\|_{L_{T x}^{r}}+\sum_{j=1}^{\infty}\left\|\Delta_{j} u\right\|_{L_{T x}^{r}} \leq C(r) T^{\nu}\left(\left\|u_{0}\right\|_{\dot{B}_{2, q}^{s_{k}}}+\|u\|_{E_{q, T}}^{k}\right) .
$$

We first consider the high frequencies. Let $r>4$, then there always exists $q_{1} \geq r$, such that $q_{1} \in[2,+\infty]$ with $\frac{1}{r}+\frac{1}{2 q_{1}} \leq \frac{1}{4}$ and $s_{k}-\frac{1}{2}+\frac{1}{r}+\frac{4}{q_{1}}>0$.

Indeed, we can clearly find such a $q_{1}$ as long as the set $\left(\frac{3}{4(k-1)}-\frac{1}{4 r}, \min \left(\frac{1}{r}, \frac{1}{2}-\right.\right.$ $\left.\frac{2}{r}\right)$ ) is nonempty. The conditions

$$
\frac{3}{4(k-1)}-\frac{1}{4 r}<\frac{1}{r} \Leftrightarrow r<\frac{5}{3}(k-1)
$$

and

$$
\frac{3}{4(k-1)}-\frac{1}{4 r}<\frac{1}{2}-\frac{2}{r} \Leftrightarrow r>\frac{7(k-1)}{2 k-5} .
$$


Using Lemma 4.1 and Hölder inequality, we get

$$
\sum_{j=1}^{\infty}\left\|\Delta_{j} u\right\|_{L_{x}^{r} L_{T}^{q_{1}}}=\left\|2^{-j\left(s_{k}-\frac{1}{2}+\frac{1}{r}+\frac{4}{q_{1}}\right)} \alpha_{j}\right\|_{l^{1}(j \geq 1)} \leq C(r)\left(\left\|u_{0}\right\|_{\dot{B}_{2, q}^{s_{k}}}+\|u\|_{E_{q, T}}^{k}\right) .
$$

Using Hölder inequality and (4.6), we obtain

$$
\sum_{j=1}^{\infty}\left\|\Delta_{j} u\right\|_{L_{T x}^{r}} \leq T^{\nu} \sum_{j=1}^{\infty}\left\|\Delta_{j} u\right\|_{L_{x}^{r} L_{T}^{q_{1}}} \leq C(r) T^{\nu}\left(\left\|u_{0}\right\|_{\dot{B}_{2, q}^{s_{k}}}+\|u\|_{E_{q, T}}^{k}\right) .
$$

Here $\nu=\frac{1}{r}-\frac{1}{q}>0$.

Now we consider the low frequencies term. Let $r \in\left[4, \frac{5}{3}(k-1)\right)$, set $q_{2}=\infty$, then we get $\frac{1}{r}+\frac{1}{\infty} \leq \frac{1}{4}$ and $s_{k}-\frac{1}{2}+\frac{1}{r}+\frac{4}{\infty}<0$ as long as $r \geq 4$ and $r>\frac{k-1}{3}$. From Lemma 4.1 again gives

$$
\sum_{j=-\infty}^{0}\left\|\Delta_{j} u\right\|_{L_{x}^{r} L_{T}^{q_{2}}}=\left\|2^{-j\left(s_{k}-\frac{1}{2}+\frac{1}{r}+\frac{4}{q_{2}}\right)} \alpha_{j}\right\|_{l^{1}(j \leq 0)} \leq C(r)\left(\left\|u_{0}\right\|_{\dot{B}_{2, q}^{s_{k}}}+\|u\|_{E_{q, T}}^{k}\right) .
$$

By Hölder inequality yields

$$
\sum_{j=-\infty}^{0}\left\|\Delta_{j} u\right\|_{L_{T x}^{r}} \leq T^{\nu} \sum_{j=-\infty}^{0}\left\|\Delta_{j} u\right\|_{L_{x}^{r} L_{T}^{q_{2}}} \leq C(r) T^{\nu}\left(\left\|u_{0}\right\|_{\dot{B}_{2, q}^{s_{k}}}+\|u\|_{E_{q, T}}^{k}\right) .
$$

Here $\nu=\frac{1}{r}>0$.

Thus, Combining (4.7) and (4.9) gives the proof of (4.5). The proof of Lemma 4.2 is completed.

Lemma 4.3 Assume that $u_{0} \in \dot{B}_{2, q}^{s_{k}}$ and $a b \leq 0(b \neq 0)$, where $1 \leq q<\infty$. Then for any $\varepsilon>0$, there exists $T=T\left(\left\|u_{0}\right\|_{\dot{B}_{2, q}^{s_{k}}}, \varepsilon\right)$ small enough such that

$$
\left\|S(t) u_{0}\right\|_{E_{q, T}} \leq \varepsilon
$$

Proof. From Lemma 2.6, for any $T$ and $u_{0} \in \dot{B}_{2, q}^{s_{k}}$, we get

$$
\left\|S(t) u_{0}\right\|_{N_{i, q, T}} \leq C\left\|u_{0}\right\|_{\dot{B}_{2, q}^{s_{k}}} . \quad i=1,2 .
$$

Since $Z(\mathbb{R})$ is dense in $\dot{B}_{2, q}^{s_{k}}$, there exists $v_{0} \in Z(\mathbb{R})$ such that for any $\varepsilon>0$

$$
\left\|u_{0}-v_{0}\right\|_{\dot{B}_{2, q}^{s}} \leq \frac{\varepsilon}{4 C}
$$


Using Lemma 2.3 yields

$$
\begin{aligned}
& \left\|S(t) v_{0}\right\|_{N_{i, q, T}}=\left\|2^{j s_{k}} 2^{j\left(\frac{1}{p_{i}}+\frac{4}{q_{i}}-\frac{1}{2}\right)}\right\| \Delta_{j} S(t) v_{0}\left\|_{L_{x}^{p_{i}} L_{T}^{q_{i}}}\right\|_{l^{q}(\mathbb{Z})} \\
& \leq C T^{\frac{1}{q_{i}}}\left\|2^{j s_{k}} 2^{j\left(\frac{1}{p_{i}}+\frac{4}{q_{i}}-\frac{1}{2}\right)}\right\| \Delta_{j} S(t) v_{0}\left\|_{L_{x}^{p_{i}} L_{T}^{\infty}}\right\|_{l^{q}(\mathbb{Z})} \\
& \leq C T^{\frac{1}{q_{i}}}\left\|2^{j s_{k}} 2^{j \frac{4}{q_{i}}}\right\| \Delta_{j} v_{0}\left\|_{L^{2}}\right\|_{l^{q}(\mathbb{Z})} \\
& =C T^{\frac{1}{q_{i}}}\left\|v_{0}\right\|_{\dot{B}_{2, q} s_{k}+\frac{4}{q_{i}}} \text {. }
\end{aligned}
$$

Since $v_{0} \in Z(\mathbb{R}) \subset \dot{B}_{2, q}^{s_{k}+\frac{4}{q_{i}}}$, then we obtain

$$
\left\|S(t) v_{0}\right\|_{N_{i, q, T}} \leq C T^{\frac{1}{q_{i}}} .
$$

Choosing $T>0$ small enough such that $C T^{\frac{1}{q_{i}}} \leq \frac{\varepsilon}{4}, \quad i=1$, 2 . So we get

$$
\begin{aligned}
\left\|S(t) u_{0}\right\|_{E_{q, T}}= & \left\|S(t) u_{0}\right\|_{N_{1, q, T}}+\left\|S(t) u_{0}\right\|_{N_{2, q, T}} \\
\leq & \left\|S(t)\left(u_{0}-v_{0}\right)\right\|_{N_{1, q, T}}+\left\|S(t) v_{0}\right\|_{N_{1, q, T}}+ \\
& \left\|S(t)\left(u_{0}-v_{0}\right)\right\|_{N_{2, q, T}}+\left\|S(t) v_{0}\right\|_{N_{2, q, T}} \\
\leq & \frac{\varepsilon}{4}+\frac{\varepsilon}{4}+\frac{\varepsilon}{4}+\frac{\varepsilon}{4} \leq \varepsilon .
\end{aligned}
$$

This proves Lemma 4.3.

Lemma 4.4 Assume that $u_{0} \in B_{2, q}^{s}, 1 \leq q \leq \infty, a b \leq 0(b \neq 0)$ and $s>s_{k}$, then for $s>s^{\prime}>s_{k}$, there exists $\eta_{i}>0(i=1,2)$ such that

$$
\left\|S(t) u_{0}\right\|_{N_{i, q, T}} \leq C T^{\eta_{i}}\left\|u_{0}\right\|_{B_{2, q}^{s^{\prime}},}, i=1,2 .
$$

Proof. For $q_{i} \in[2, \infty]$, choosing $r_{i}>q_{i}$, such that $s^{\prime}-s_{k} \geq \frac{4}{q_{i}}-\frac{4}{r_{i}}$. Using Hölder inequality, Lemma 2.3 and $B_{2, q}^{s^{\prime}} \hookrightarrow \dot{B}_{2, q}^{s_{k}+\frac{4}{q_{i}}-\frac{4}{r_{i}}}$, we obtain

$$
\begin{aligned}
& \left\|S(t) u_{0}\right\|_{N_{i, q, T}}=\left\|2^{j s_{k}} 2^{j\left(\frac{1}{p_{i}}+\frac{4}{q_{i}}-\frac{1}{2}\right)}\right\| \Delta_{j} S(t) u_{0}\left\|_{L_{x}^{p_{i}} L_{T}^{q_{i}}}\right\|_{l^{q}(\mathbb{Z})} \\
& =2 T^{\frac{1}{q_{i}}-\frac{1}{r_{i}}}\left\|2^{j s_{k}} 2^{j\left(\frac{4}{q_{i}}-\frac{4}{r_{i}}\right)} 2^{j\left(\frac{1}{p_{i}}+\frac{4}{q_{i}}-\frac{1}{2}\right)} \Delta_{j} S(t) u_{0}\right\|_{L_{x}^{p_{i}} L_{T}^{r_{i}} \|_{l q}(\mathbb{Z})} \\
& \leq C T^{\frac{1}{q_{i}}-\frac{1}{r_{i}}}\left\|2^{j\left(s_{k}+\frac{4}{q_{i}}-\frac{4}{r_{i}}\right)}\right\| \Delta_{j} u_{0}\left\|_{L^{2}}\right\|_{l^{q}(\mathbb{Z})} \\
& =C T^{\frac{1}{q_{i}}-\frac{1}{r_{i}}}\left\|u_{0}\right\|_{\dot{B}^{s_{k}}+\frac{4}{q_{i}}-\frac{4}{r_{i}}} \\
& \leq C T^{\frac{1}{q_{i}}-\frac{1}{r_{i}}}\left\|u_{0}\right\|_{B_{2, q}^{s^{\prime}}} \text {. }
\end{aligned}
$$

Choosing $\eta_{i}=\frac{1}{q_{i}}-\frac{1}{r_{i}}$, then proof of Lemma 4.4 is finished.

In what follows, we give main theorem. 
Theorem 4.1 Suppose that $k \geq 5, k \in \mathbb{N}$ and $a b \leq 0(b \neq 0)$, suppose furthermore that there exists $\delta=\delta(k)>0$ such that for any $u_{0} \in \dot{B}_{2, \infty}^{s_{k}}$ with $\left\|u_{0}\right\|_{\dot{B}_{2, \infty}^{s_{k}}} \leq \delta$. Then the IVP (1.1)-(1.2) has a unique solution $u=u(x, t)$ on the domain $\mathbb{R} \times \mathbb{R}$ and the solution satisfies the following properties

$$
\begin{gathered}
u \in C\left(\mathbb{R} ; \dot{B}_{2, \infty}^{s_{k}}\right) . \\
N_{1, \infty}+N_{2, \infty} \leq 4 C \delta
\end{gathered}
$$

and

$$
u(t) \rightarrow u_{0}
$$

in $\mathbb{S}^{\prime}(\mathbb{R})$ as $t \rightarrow 0$.

Moreover, for any $T<\infty$,

$$
u \in L_{T x}^{r}, r \in \begin{cases}\left(\frac{7(k-1)}{2 k-5}, \frac{5(k-1)}{3}\right), & 5 \leq k \leq 13 \\ \left(\frac{k-1}{3}, \frac{5(k-1)}{3}\right), & k \geq 13\end{cases}
$$

and the mapping $u_{0} \rightarrow u$ is lipschitz continous from $\left\{u_{0} \in \dot{B}_{2, \infty}^{s_{k}} \mid\left\|u_{0}\right\|_{\dot{B}_{2, \infty}^{s_{k}}} \leq \delta\right\}$ into the space defined by (4.12)-(4.14).

Proof. For any fixed $M>0$, we define the function space

$$
X_{M} \triangleq\left\{u \mid u-S(t) u_{0} \in C\left(\mathbb{R} ; \dot{B}_{2, \infty}^{s_{k}}\right) \bigcap E_{\infty},\left\|u-S(t) u_{0}\right\|_{L_{t}^{\infty} \dot{B}_{2, \infty}^{s_{k}}}+\|u\|_{E_{\infty}} \leq M\right\}
$$

equipped with the metric

$$
d\left(u_{1}, u_{2}\right)=\left\|u_{1}-u_{2}\right\|_{L_{t}^{\infty} \dot{B}_{2, \infty}^{s_{k}}}+\left\|u_{1}-u_{2}\right\|_{E_{\infty}} .
$$

Obviously, $\left(X_{M}, d\right)$ is a complete metric space.

For any $u \in X_{M}$, Lemma 2.6 and 3.1 gives

$$
\begin{gathered}
\left\|\Phi(u)-S(t) u_{0}\right\|_{L_{t}^{\infty} \dot{B}_{2, \infty}^{s_{k}}}+\|\Phi(u)\|_{E_{\infty}} \\
\leq\left\|\int_{0}^{t} S(t-\tau) \partial_{x} u^{k}(\tau) d \tau\right\|_{L_{t}^{\infty} \dot{B}_{2, \infty}^{s_{k}}}+\left\|S(t) u_{0}\right\|_{E_{\infty}}+\left\|\int_{0}^{t} S(t-\tau) \partial_{x} u^{k}(\tau) d \tau\right\|_{E_{\infty}} \\
\leq C\|u\|_{E_{\infty}}^{k}+C\left\|u_{0}\right\|_{\dot{B}_{2, \infty}^{s_{k}}} .
\end{gathered}
$$

Choosing $M=(4 C)^{-\frac{1}{k-1}}$ and $\delta=(4 C)^{-\frac{k}{k-1}}$, since $\left\|u_{0}\right\|_{\dot{B}_{2, \infty}^{s_{k}}} \leq \delta$, then from (4.16), we get

$$
\left\|\Phi(u)-S(t) u_{0}\right\|_{L_{t}^{\infty} \dot{B}_{2, \infty}^{s_{k}}}+\|\Phi(u)\|_{E_{\infty}} \leq M .
$$

So $\Phi(u) \in X_{M}$. 
For $u_{1}, u_{2} \in X_{M}$, using Lemma 3.2 gives

$$
\begin{gathered}
d\left(u_{1}, u_{2}\right)=\left\|\Phi\left(u_{1}\right)-\Phi\left(u_{2}\right)\right\|_{L_{t}^{\infty} \dot{B}_{2, \infty}^{s_{k}}}+\left\|\Phi\left(u_{1}\right)-\Phi\left(u_{2}\right)\right\|_{E_{\infty}} \\
\leq\left\|\int_{0}^{t} S(t-\tau) \partial_{x}\left(u_{1}^{k}-u_{2}^{k}\right)(\tau) d \tau\right\|_{L_{t}^{\infty} \dot{B}_{2, \infty}^{s_{k}}}+\left\|\int_{0}^{t} S(t-\tau) \partial_{x}\left(u_{1}^{k}-u_{2}^{k}\right)(\tau) d \tau\right\|_{E_{\infty}} \\
\leq C\left\|u_{1}-u_{2}\right\|_{E_{\infty}}\left(\left\|u_{1}\right\|_{E_{\infty}}^{k-1}+\left\|u_{2}\right\|_{E_{\infty}}^{k-1}\right) \\
\leq \frac{1}{2}\left\|u_{1}-u_{2}\right\|_{E_{\infty}} \leq \frac{1}{2} d\left(u_{1}, u_{2}\right) .
\end{gathered}
$$

So $\Phi$ is a strict contractive mapping on $X_{M}$.

Using Banach fixed point theorem, there exists a unique $u \in X_{M}$ satisfying (4.12) and (4.13).

In what follows, we prove (4.14) and (4.15). Since $u-S(t) u_{0} \in C\left(\mathbb{R} ; \dot{B}_{2, \infty}^{s_{k}}\right)$ and the continuity of the group $S(t)$ in $\mathbb{S}^{\prime}(\mathbb{R})$ yields (4.14). Lemma 4.2 assure that (4.15) holds. The proof of theorem 4.1 is completed.

Theorem 4.2 Suppose that $k \geq 5, k \in N$ and $a b \leq 0(b \neq 0)$, suppose furthermore that $u_{0} \in \dot{B}_{2, q}^{s_{k}}(\mathbb{R})$ and $q \in[1, \infty)$.

(1) Then there exists a positive constant $T=T\left(\left\|u_{0}\right\|_{\dot{B}_{2, q}^{s_{k}}}\right)$ such that the IVP (1.1)-(1.2) has a unique solution $u=u(x, t)$ on the strip $\mathbb{R} \times[-T, T]$ and the solution satisfies the following properties

$$
u \in C\left([-T, T] ; \dot{B}_{2, q}^{s_{k}}\right) \bigcap E_{q, T} .
$$

Moreover, for all $T<+\infty$

$$
u \in L_{T x}^{r}, r \in \begin{cases}\left(\frac{7(k-1)}{2 k-5}, \frac{5(k-1)}{3}\right), & 5 \leq k \leq 13 \\ \left(\frac{k-1}{3}, \frac{5(k-1)}{3}\right), & k \geq 13\end{cases}
$$

and the mapping $u_{0} \longmapsto u(t)$ from $\dot{B}_{2, q}^{s_{k}}(\mathbb{R})$ into the space defined by $(4.17)$ is locally Lipschitz.

(2) If $\left\|u_{0}\right\|_{\dot{B}_{2, \infty}^{s_{k}}} \leq \delta$ (given in the statement of theorem 4.1 ), then $u$ is a global solution of IVP (1.1)-(1.2) and satisfies

$$
u \in C\left(\mathbb{R} ; \dot{B}_{2, q}^{s_{k}}\right) \bigcap L^{\infty}\left(\mathbb{R} ; \dot{B}_{2, q}^{s_{k}}\right) .
$$

Moreover, for all $T<+\infty,(4.18)$ also holds. The mapping $u_{0} \longmapsto u(t)$ from $\dot{B}_{2, q}^{s_{k}}(\mathbb{R})$ into the space defined by (4.19) is locally Lipschitz.

Proof. (1) For fixed $M, T>0$, we define the metric space

$$
X_{T}^{M}=\left\{u \in C\left([-T, T] ; \dot{B}_{2, q}^{s_{k}}\right) \bigcap E_{q, T} \mid\left\|u-S(t) u_{0}\right\|_{L_{T}^{\infty} \dot{B}_{2, q}^{s_{k}}}+\|u\|_{E_{q, T}} \leq M\right\},
$$




$$
d\left(u_{1}, u_{2}\right)=\left\|u_{1}-u_{2}\right\|_{L_{T}^{\infty} \dot{B}_{2, q}^{s_{k}}}+\left\|u_{1}-u_{2}\right\|_{E_{q, T}} .
$$

Obviously, $\left(X_{T}^{M}, d\right)$ is a complete metric space.

For any $u \in X_{T}^{M}$, using Lemma 3.1, we get

$$
\begin{gathered}
\left\|\Phi(u)-S(t) u_{0}\right\|_{L_{T}^{\infty} \dot{B}_{2, q}^{s_{k}}} \leq\left\|\int_{0}^{t} S(t-\tau) \partial_{x} u^{k}(\tau) d \tau\right\|_{L_{T}^{\infty} \dot{B}_{2, q}^{k}} \\
\leq C\|u\|_{E_{q, T}}\|u\|_{E_{\infty, T}}^{k-1} \leq C M^{k} .
\end{gathered}
$$

From Lemma 2.6, 3.1 and 4.3, we obtain

$$
\begin{aligned}
\|\Phi(u)\|_{E_{q, T}} & =\left\|S(t) u_{0} \pm i \int_{0}^{t} S(t-\tau) \partial_{x} u^{k}(\tau) d \tau\right\|_{E_{q, T}} \\
& \leq\left\|S(t) u_{0}\right\|_{E_{q, T}}+\left\|\int_{0}^{t} S(\tau) d \tau\right\|_{E_{q, T}} \\
& \leq\left\|S(t) u_{0}\right\|_{E_{q, T}}+C\|u\|_{E_{q, T}}\|u\|_{E_{\infty, T}}^{k-1} \\
& \leq \varepsilon+c M^{k}
\end{aligned}
$$

Combining (4.20) and (4.21) yields

$$
\left\|\Phi(u)-S(t) u_{0}\right\|_{L_{T}^{\infty} \dot{B}_{2, q}^{s_{k}}}+\|\Phi(u)\|_{E_{q, T}} \leq \varepsilon+C M^{k} .
$$

Now choosing $M>0$ small enough such that

$$
C M^{k-1} \leq \frac{1}{4}
$$

Let $0<\varepsilon \leq \frac{1}{2} M$, then we have

$$
\left\|\Phi(u)-S(t) u_{0}\right\|_{L_{T}^{\infty} \dot{B}_{2, q}^{s_{k}}}+\|\Phi(u)\|_{E_{q, T}} \leq M,
$$

So $\Phi$ maps $X_{T}^{M}$ into $X_{T}^{M}$.

We now show that $\Phi$ is a strict contractive map. For any $u_{1}, u_{2} \in X_{T}^{M}$, it follows from Lemma 3.2 that

$$
\begin{aligned}
& \left\|\Phi\left(u_{1}\right)-\Phi\left(u_{2}\right)\right\|_{L_{T}^{\infty} \dot{B}_{2, q}^{s_{k}}}+\left\|\Phi\left(u_{1}\right)-\Phi\left(u_{2}\right)\right\|_{E_{q, T}} \\
& \quad \leq C\left\|u_{1}-u_{2}\right\|_{E_{q, T}}\left(\left\|u_{1}\right\|_{E_{\infty, T}}^{k-1}+\left\|u_{2}\right\|_{E_{\infty, T}}^{k_{1}}\right) \\
& \quad \leq 2 C M^{k-1}\left\|u_{1}-u_{2}\right\|_{E_{q, T}} \\
& \quad \leq \frac{1}{2}\left\|u_{1}-u_{2}\right\|_{E_{q, T}} .
\end{aligned}
$$

From the contraction mapping principle, the IVP (1.1)-(1.2) has a unique solution $u(x, t)$ on the strip $\mathbb{R} \times[-T, T]$ and the solution satisfies the properties 
(4.17) and (4.18). From the above proof, we know that the map $u_{0} \longmapsto u(t)$ is lipschitz continuous. we have proved the first part in Theorem 4.2.

(2) Let $u_{0} \in \dot{B}_{2, q}^{s_{k}}(\mathbb{R}), q \in[1, \infty)$ with $\left\|u_{0}\right\|_{\dot{B}_{2, \infty}^{s_{k}}} \leq \delta$, (recalling that $\dot{B}_{2, q}^{s_{k}} \hookrightarrow$ $\left.\dot{B}_{2, \infty}^{s_{k}}\right)$. By virtue of Theorem 4.1, $\left\{\omega \in E_{\infty} \mid\|\omega\|_{E_{\infty}} \leq 4 C \delta\right\}$ is stable by $\Phi$.

For any fixed $M>0$, we define metric space

$$
X_{M}=\left\{u \in C\left(\mathbb{R} ; \dot{B}_{2, q}^{s_{k}}\right) \bigcap E_{q} \mid\|u\|_{E_{\infty}} \leq M\right\}
$$

equipped with the metric

$$
d\left(u_{1}, u_{2}\right)=\left\|u_{1}-u_{2}\right\|_{L_{t}^{\infty} \dot{B}_{2, q}^{s_{k}}}+\left\|u_{1}-u_{2}\right\|_{E_{q}}
$$

In what follows, we show that $\Phi$ maps $X_{M}$ into $X_{M}$. For $u \in X_{M}$, By Lemma 2.6 and 3.1, we obtain

$$
\begin{aligned}
\|\Phi(u)\|_{E_{\infty}} & \leq\left\|S(t) u_{0}\right\|_{E_{\infty}}+\left\|\int_{0}^{t} S(t-\tau) \partial_{x}^{k}(\tau) d \tau\right\|_{E_{\infty}} \\
& \leq\left\|u_{0}\right\|_{\dot{B}_{2, \infty}^{s_{k}}}+C\|u\|_{E_{\infty}}^{k} \\
& \leq C \delta+c M^{k} .
\end{aligned}
$$

Choosing $M=(4 C)^{-\frac{1}{k-1}}$ and $\delta=(2 C)^{-1}(4 C)^{-\frac{1}{k-1}}$, then from the above inequality, we get

$$
\|\Phi(u)\|_{E_{\infty}} \leq M
$$

For $u_{1}, u_{2} \in X_{M}$, it follows from Lemma 3.2 that

$$
\begin{aligned}
d\left(\Phi\left(u_{1}\right), \Phi\left(u_{2}\right)\right) & =\left\|\Phi\left(u_{1}\right)-\Phi\left(u_{2}\right)\right\|_{L_{t}^{\infty} \dot{B}^{s_{k}}}+\left\|\Phi\left(u_{1}\right)-\Phi\left(u_{2}\right)\right\|_{E_{q}} \\
& \leq C\left\|u_{1}-u_{2}\right\|_{E_{q}}\left(\left\|u_{1}\right\|_{E_{\infty}}^{k-1}+\left\|u_{2}\right\|_{E_{\infty}}^{k-1}\right) \\
& \leq 2 C M^{k-1}\left\|u_{1}-u_{2}\right\|_{E_{q}} \\
& \leq \frac{1}{2} d\left(u_{1}, u_{2}\right) .
\end{aligned}
$$

So $\Phi$ is a strict contractive map on $X_{M}$.

From Banach fixed Theorem, the IVP (1.1)-(1.2) has a unique solution $u(x, t)$ and the solution satisfies the properties (4.19). From the above proof, we know that the map $u_{0} \longmapsto u(t)$ is lipschitz continuous. From Lemma 4.2, we know that (4.18) also holds. The Theorem 4.2 is proved.

Theorem 4.3 Assume that $a b \leq 0(b \neq 0), k \geq 5, k \in \mathbb{N}$ and $s>s^{\prime}>s_{k}$, suppose further $u_{0} \in B_{2, q}^{s}$ and $1 \leq q<\infty$. 
(1) Then there exists a positive constant $T=T\left(\left\|u_{0}\right\|_{B_{2, q}^{s^{\prime}}}\right)$ such that the IVP (1.1)-(1.2) has a unique solution $u=u(x, t)$ on the strip $\mathbb{R} \times[-T, T]$ and the solution satisfies the following properties

$$
u \in C\left([-T, T] ; B_{2, q}^{s}\right) \bigcap E_{q, T}
$$

and

$$
D_{x}^{s-s_{k}} u \in L_{T x}^{r}, r \in\left\{\begin{array}{l}
\left(\frac{7(k-1)}{2 k-5}, \frac{5(k-1)}{3}\right), \quad 5 \leq k \leq 13 \\
\left(\frac{k-1}{3}, \frac{5(k-1)}{3}\right), \quad k \geq 13
\end{array}\right.
$$

and the mapping $u_{0} \longmapsto u(t)$ from $B_{2, q}^{s}(\mathbb{R})$ into the space defined by (4.22) is locally Lipschitz.

(2) If $\left\|u_{0}\right\|_{\dot{B}_{2, \infty}^{s_{k}}} \leq \delta$ (given by Theorem 4.1), then $u$ is a global solution and satisfies the properties

$$
u \in L^{\infty}\left(\mathbb{R} ; B_{2, q}^{s}\right) .
$$

Proof. For fixed $M, T>0$, define metric space $\left(X_{T}^{M}, d\right)$

$$
\begin{gathered}
X_{T}^{M}=\left\{u \in C\left([-T, T] ; B_{2, q}^{s}\right) \bigcap E_{q, T} \bigcap L_{T x}^{k} \mid\|u\|_{E_{q, T}}+\|u\|_{L_{T x}^{k}}\right. \\
\left.+\lambda\left(\|u\|_{L_{T}^{\infty} B_{2, q}^{s}}+\|u\|_{N_{2, q, T}^{s}}\right) \leq M\right\}, \\
d\left(u_{1}, u_{2}\right)=\left\|u_{1}-u_{2}\right\|_{E_{q, T}}+\left\|u_{1}-u_{2}\right\|_{L_{T x}^{k}}+\lambda\left(\left\|u_{1}-u_{2}\right\|_{L_{T}^{\infty} B_{2, q}^{s}}+\left\|u_{1}-u_{2}\right\|_{N_{2, q, T}^{s}}\right),
\end{gathered}
$$

where

and

$$
\lambda=\frac{\left\|S(t) u_{0}\right\|_{E_{q, T}}}{\left\|u_{0}\right\|_{B_{2, q}^{s}}^{s}}
$$

$$
\|u\|_{N_{2, q, T}^{s}}=\left\|2^{j s} 2^{j\left(\frac{1}{p_{2}}+\frac{4}{q_{2}}-\frac{1}{2}\right)}\right\| \Delta_{j} u\left\|_{L_{x}^{p_{2}} L_{T}^{q_{2}}}\right\|_{l^{q}(\mathbb{Z})} .
$$

For any $u \in X_{T}^{M}$, using Lemma 3.1, 4.4 and $E_{q, T} \hookrightarrow E_{\infty, T}$, for $0<T \leq 1$, we obtain

$$
\begin{aligned}
\|\Phi(u)\|_{E_{q, T}} & \leq\left\|S(t) u_{0}\right\|_{E_{q, T}}+\left\|\int_{0}^{t} S(t-\tau) \partial_{x} u^{k}(\tau) d \tau\right\|_{E_{q, T}} \\
& \leq\left\|S(t) u_{0}\right\|_{E_{q, T}}+C\|u\|_{E_{q, T}}\|u\|_{E_{\infty}, T}^{k-1} \\
& \leq C\left(T^{\eta}\left\|u_{0}\right\|_{B_{2, q}^{s^{\prime}}}+\|u\|_{E_{q, T}}^{k}\right) \\
& \leq C\left(T^{\eta}\left\|u_{0}\right\|_{B_{2, q}^{s^{\prime}}}+M^{k}\right) .
\end{aligned}
$$

From Lemma 4.2 , for $0<T \leq 1$, we get

$$
\|\Phi(u)\|_{L_{T x}^{k}} \leq C\left(T^{\nu}\left\|u_{0}\right\|_{B_{2, q}^{s^{\prime}}}+\|u\|_{E_{q, T}}^{k}\right) \leq C\left(T^{\nu}\left\|u_{0}\right\|_{B_{2, q}^{s^{\prime}}}+M^{k}\right) .
$$


For $t \in \mathbb{R}, S(t)$ is a unitary group in $H^{s}(\mathbb{R})$, using Young inequality, we obtain

$$
\begin{aligned}
\left\|S_{0}(\Phi(u))\right\|_{L_{T}^{\infty} L_{x}^{2}} & \leq\left\|S_{0}\left(S(t) u_{0}\right)\right\|_{L_{T}^{\infty} L_{x}^{2}}+\left\|S_{0}\left(\int_{0}^{t} S(t-\tau) \partial_{x} u^{k}(\tau) d \tau\right)\right\|_{L_{T}^{\infty} L_{x}^{2}} \\
& \leq\left\|S_{0} u_{0}\right\|_{L^{2}}+\sup _{t \in[-T, T]} \int_{0}^{t}\left\|\partial_{x} S(t-\tau) S_{0} u^{k}(\tau)\right\|_{L_{x}^{2}} d \tau \\
& \leq\left\|u_{0}\right\|_{B_{2, q}^{s}}+C \int_{-T}^{T}\left\|S_{0}\left(u^{k}(t)\right)\right\|_{L^{2}} d t \\
& \leq\left\|u_{0}\right\|_{B_{2, q}^{s}}+C\left\|\psi_{0}\right\|_{L^{2}} \int_{-T}^{T}\left\|u^{k}(t)\right\|_{L^{1}} d t \\
& \leq\left\|u_{0}\right\|_{B_{2, q}^{s}}+C\|u\|_{L_{T x}^{k}}^{k}
\end{aligned}
$$

Using (4.27), analogous to the nonlinear estimate as those in Lemma 3.1, for $0<T \leq 1$, we can establish

$$
\begin{aligned}
\|\Phi(u)\|_{L_{T}^{\infty} B_{2, q}^{s}}+\|\Phi(u)\|_{N_{2, q, T}^{s}} & \leq\left\|S_{0}(\Phi(u))\right\|_{L_{T}^{\infty} L_{x}^{2}}+\|\Phi(u)\|_{L_{T}^{\infty} \dot{B}_{2, q}^{s}}+\|\Phi(u)\|_{N_{2, q, T}^{s}} \\
& \leq C\left(\left\|u_{0}\right\|_{B_{2, q}^{s}}+\|u\|_{L_{T x}^{k}}^{k}+\|u\|_{N_{1, \infty}, T}^{k-1}\|u\|_{N_{2, q, T}}\right) \\
& \leq C\left(\left\|u_{0}\right\|_{B_{2, q}^{s}}+\|u\|_{X_{T}^{M}}^{k}\right) \\
& \leq C\left(\left\|u_{0}\right\|_{B_{2, q}^{s}}+M^{k}\right) .
\end{aligned}
$$

For $0<T \leq 1$, Lemma 4.4 gives

$$
\lambda \leq C T^{\eta} \frac{\left\|u_{0}\right\|_{B_{2, q}^{s^{\prime}}}}{\left\|u_{0}\right\|_{B_{2, q}^{s}}} \leq C T^{\eta} \leq c,
$$

Thus

$$
\lambda\left(\|\Phi(u)\|_{L_{T}^{\infty} B_{2, q}^{s}}+\|\Phi(u)\|_{N_{2, q, T}^{s}}\right) \leq C\left(\lambda\left\|u_{0}\right\|_{B_{2, q}^{s}}+\lambda M^{k}\right) \leq C\left(T^{\eta}\left\|u_{0}\right\|_{B_{2, q}^{s^{\prime}}}+M^{k}\right) .
$$

For $0<T \leq 1$ and any $u \in X_{T}^{M}$, combining (4.25), (4.26) and (4.28) yields

$$
\|\Phi(u)\|_{X_{T}^{M}} \leq C\left(T^{\max \{\eta, \nu\}}\left\|u_{0}\right\|_{B_{2, q}^{s^{\prime}}}+M^{k}\right) .
$$

Choosing $M>0$ and $T>0$ suitably small such that

$$
C M^{k-1} \leq \frac{1}{4}
$$

and

hold, then we have

$$
T^{\max \{\eta, \nu\}} \leq \frac{M}{4 C\left\|u_{0}\right\|_{B_{2, q}^{s^{\prime}}}},
$$

$$
\|\Phi(u)\|_{X_{T}^{M}} \leq M
$$


This implies that $\Phi$ maps $X_{T}^{M}$ into $X_{T}^{M}$.

In what follows, we will show that $\Phi$ is a strictly mapping on $X_{T}^{M}$. For any $u_{1}, u_{2} \in X_{T}^{M}$, using the same way to obtain (4.29), we get

$$
\left\|\Phi\left(u_{1}\right)-\Phi\left(u_{2}\right)\right\|_{X_{T}^{M}} \leq C\left(\left\|u_{1}\right\|_{X_{T}^{M}}^{k-1}+\left\|u_{2}\right\|_{X_{T}^{M}}^{k-1}\right)\left\|u_{1}-u_{2}\right\|_{X_{T}^{M}} .
$$

If $M$ satisfies $C M^{k-1} \leq \frac{1}{4}$, then we have

$$
\left\|\Phi\left(u_{1}\right)-\Phi\left(u_{2}\right)\right\|_{X_{T}^{M}} \leq \frac{1}{2}\left\|u_{1}-u_{2}\right\|_{X_{T}^{M}} .
$$

From the contraction mapping principle, the IVP (1.1)-(1.2) has a unique solution $u(x, t)$ on the strip $\mathbb{R} \times[-T, T]$ and the solution satisfies the property (4.22). From the above proof, we know that the map $u_{0} \longmapsto u(t)$ is lipschitz continuous. From $L_{T}^{\infty} B_{2, q}^{s} \hookrightarrow L_{T}^{\infty} L_{x}^{2}$ and Lemma 4.2, (4.23) follows.

The proof of the second part of Theorem 4.3. By the same way to that of proof of Lemma 3.1 and Lemma 3.2, and from Theorem 4.1, $\|u\|_{E_{\infty}}^{k-1} \leq(4 C)^{-1}$ for $\left\|u_{0}\right\|_{\dot{B}_{2, \infty}^{s_{k}}} \leq \delta$, we can show that for any fixed $\theta \in[0, s]$ and $T>0$

$$
\begin{aligned}
\|u\|_{L_{T}^{\infty} \dot{B}_{2, q}^{\theta}}+\|u\|_{\dot{N}_{2, q, T}^{\theta}} & \leq C\left\|u_{0}\right\|_{\dot{B}_{2, q}^{\theta}}+C\|u\|_{E_{\infty}}^{k-1}\|u\|_{\dot{N}_{2, q, T}^{\theta}} \\
& \leq C\left\|u_{0}\right\|_{\dot{B}_{2, q}^{\theta}}+\frac{1}{2}\|u\|_{\dot{N}_{2, q, T}^{\theta}} \\
& \leq 2 C\left\|u_{0}\right\|_{\dot{B}_{2, q}^{\theta}} \leq 2 C\left\|u_{0}\right\|_{B_{2, q}^{\theta}} .
\end{aligned}
$$

Taking $\theta=0$ and $s$, respectively, this proves that $u$ can be extended for al time and $u \in L^{\infty}\left(\mathbb{R} ; B_{2, q}^{s}\right)$. This proves Theorem 4.3.

\section{SCATTERING}

In this section, we give the scattering result.

Theorem 5.1 Assume that $u_{0} \in \dot{B}_{2, q}^{s_{k}}, q \in[1, \infty)$ and $a b \leq 0(b \neq 0)$, and the initial value satisfy the small assumption of Theorem 4.1. Let $u(x, t)$ be the solution of the IVP (1.1)- (1.2). Then there exists $\left(u_{0}^{-}, u_{0}^{+}\right) \in \dot{B}_{2, q}^{s_{k}} \times \dot{B}_{2, q}^{s_{k}}$ such that

$$
\lim _{t \rightarrow+\infty}\left\|u(t)-S(t) u_{0}^{+}\right\|=0 ; \quad \lim _{t \rightarrow-\infty}\left\|u(t)-S(t) u_{0}^{-}\right\|=0 .
$$

Proof. The proof of essentially follows from the proof of Theorem 2.2 in [15]. From the proof of the second part of Theorem 4.2. we obtain $u \in C\left(\mathbb{R} ; \dot{B}_{2, q}^{s_{k}}\right)$ and

$$
\left\|2^{j s_{k}} 2^{j \frac{3}{8}}\right\| \Delta_{j}\left(u^{k}\right)\left\|_{L_{x}^{\frac{8}{7}} L_{t}^{\frac{4}{3}}}\right\|_{l^{q}(\mathbb{Z})}<+\infty .
$$


Therefore, for any fixed $n \in \mathbb{N}$, there exists $j_{n}>0$ such that

$$
\left\|2^{j s_{k}} 2^{j \frac{3}{8}}\right\| \Delta_{j}\left(u^{k}\right)\left\|_{L_{x}^{\frac{8}{3}} L_{t}^{\frac{4}{3}}}\right\|_{l q}\left(|j| \geq j_{n}\right) \leq \frac{1}{n}
$$

and there exists $T_{n}$ such that

$$
\left\|2^{j s_{k}} 2^{j \frac{3}{8}}\right\| \Delta_{j}\left(u^{k}\right)\left\|_{L_{x}^{\frac{8}{7}} L_{\left[T_{n},+\infty\right)}^{\frac{4}{3}}}\right\|_{l^{q}\left(|j| \leq j_{n}\right)}<\frac{1}{n} .
$$

Now by taking $u_{L, n}(t)=S\left(t-T_{n}\right) u\left(T_{n}\right)=S(t) u_{L, n}(0)$, combining (5.1) and (5.2) yields

$$
\begin{aligned}
\sup _{t \geq T_{n}}\left\|u(t)-u_{L, n}(t)\right\|_{\dot{B}_{2, q}^{s_{k}} \leq} \leq & \left\|2^{j s_{k}} 2^{j \frac{3}{8}}\right\| \Delta_{j}\left(u^{k}\right)\left\|_{L_{x}^{\frac{8}{7}} L_{\left[T_{n},+\infty\right)}^{\frac{4}{3}}}\right\|_{l^{q}(\mathbb{Z})} \\
\leq & \left\|2^{j s_{k}} 2^{j \frac{3}{8}}\right\| \Delta_{j}\left(u^{k}\right) \|_{L_{x}^{\frac{8}{7}} L_{\left[T_{n},+\infty\right)}^{\frac{4}{3}}\|\|_{l^{q}\left(|j| \leq j_{n}\right)}} \\
& +\left\|2^{j s_{k}} 2^{j \frac{3}{8}}\right\| \Delta_{j}\left(u^{k}\right)\left\|_{L_{x}^{\frac{8}{7}} L_{\left[T_{n},+\infty\right)}^{\frac{4}{3}}}\right\|_{l^{q}\left(|j| \geq j_{n}\right)} \\
\leq & \frac{2 C}{n} .
\end{aligned}
$$

Since $S(t)$ is a unitary group in $\dot{B}_{2, q}^{s_{k}}$, for $t \geq T_{n}, m_{1} \geq n$ and $m_{2} \geq n$, we obtain

$$
\left\|u_{L, m_{1}}(0)-u_{L, m_{2}}(0)\right\|_{\dot{B}_{2, q}^{s_{k}}}=\left\|u_{L, m_{1}}(t)-u_{L, m_{2}}(t)\right\|_{\dot{B}_{2, q}^{s_{k}}} \leq \frac{4 C}{n} .
$$

Thus, $u_{L, n}(0)$ is a Cauchy sequence in $\dot{B}_{2, q}^{s_{k}}$. Let $u_{0}^{+}$be the limit of Cauchy sequence $u_{L, n}(0)$. From (5.3), we obtain

$$
\lim _{t \rightarrow+\infty}\left\|u(t)-S(t) u_{0}^{+}\right\|=0 .
$$

In the same way, we can show that

$$
\lim _{t \rightarrow-\infty}\left\|u(t)-S(t) u_{0}^{-}\right\|=0 .
$$

Then the proof of Theorem 5.1 is completed.

Acknowledgements. This work was supported in part by the Research Initiation Project for High-level Talents (201031) of North China University of Water Resources and Electric Power.

\section{REFERENCES}

[1] T. Cazenave, Semilinear Schrödinger equations, Courant Lecture Notes in Mathematics, 2003.

[2] K. B. Dysthe, Note on a modification to the nonlinear Schrödinger equation for application to deep water waves, Proc. R. Soc. Lond. Ser. A 369 (1979), 105-114. 
[3] J. Ginibre and G. Velo, On the class of nonlinear Schrödinger equations, J. Func. Anal. 32 (1979), 1-71.

[4] J. Ginibre and G. Velo, The global Cauchy problem for the nonlinear Schrödinger equation revisited, Ann. Inst. Henri Poincaré, Analyse non linéaire 2 (1985), 309-327.

[5] C. C. Hao, L. Hsiao and B. X. Wang, Well-posedness for the fourth order nonlinear Schrödinger equations, J. Math. Anal. Appl. 320 (2006), 246-265.

[6] C. C. Hao, L. Hsiao and B. X. Wang, Well-posedness of Cauchy problem for the fourth order nonlinear Schrödinger equations in multi-dimensional spaces, J. Math. Anal. Appl. 328 (2007), 58-83.

[7] A. Hasegawa and Y. Kodama, Solitons in Optical Communications, Oxford University Press, London, 1995.

[8] Z. H. Huo and Y. L. Jia, The Cauchy problem for the fourth-order nonlinear Schrödinger equation related to the vortex filament, J. Differential Equations 214 (2005), 1-35.

[9] V. I. Karpman, Stabilization of soliton instability by higher-order dispersion: Fourth-order nonlinear Schrödinger type equations, Phys. Rev. 53 (1996), 1336-1339.

[10] V. I. Karpman, Radiation of solitons described by a high-order cubic nonlinear Schrödinger equation, Physical Review E 62 (4) (2000), 5678-5687.

[11] T. Kato, On nonlinear Schrödinger equations, Ann. Inst. Henri Poincaré, Phys. Theor. 46 (1987), 113-129.

[12] C. E. Kenig, G. Ponce and L. Vega, Oscilatory integrals and regularity of dispersive equations, Indiana Univ. Math. J. 40 (1991), 33-69.

[13] C. E. Kenig, G. Ponce and L. Vega, Smoothing effects and local existence theory for the generalized nonlinear Schrödinger equations, Invent. Math. 134 (1998), 489-545.

[14] C. E. Kenig, G. Ponce and L. Vega, On the ill-posedness of some canonical dispersive equations, Duke math. J. 106 (2001), 617-633.

[15] H. Lindblad and C. D. Sogge, On existence and scattering with minimal regularity for semilinear wave equations, J. Funct. Anal. 130 (1995), 357-426.

[16] L. Molinet and F. Ribaud, it On the Cauchy problem for the generalized Korteweg-de Vries equation, Comm. Partial Differential Equations 28 (2003), 2065-2091.

[17] L. Molinet and F. Ribaud, Well-posedness results for the generalized Benjamin-Ono equation with small initial data, J. Math. Pure Appl. 83 (2004), 277-311.

[18] T. Ogawa and Y. Tsutsumi, Blow-up solutions for the nonlinear Schrödinger equation with quartic potential and periodic boundary conditions, Lect. Notes Math. 1450 (1989), 236-251

[19] F. Planchon, Self-similar solutions and semi-linear wave equations in Besov spaces, J. Math. Pure Appl. 79 (2000), 809-820.

[20] F. Planchon, On the Cauchy problem in Besov spaces for a non-linear Schrödinger equation, Commun. Contemp. Math. 2 (2000), 243-254.

[21] F. Planchon, Dispersive estimates and the 2D cubic Schrödinger equation, J. Anal. Math. 86 (2002), 319-334.

[22] J. Segata, Well-posedness for the fourth-order nonlinear Schrodinger-type equation related to the vortex filament, Diff. Int. Eqns. 16 (2003), 841-864. 
[23] C. Sulem and P. Sulem, The Nonlinear Schrödinger Equation Self-Focusing and Wave Collapse, Appl. Math. Sci. 139, Springer-Verlag, New York, 1999.

[24] N. Yajima and J. Satsuma, Soliton solutions in a diatomic lattice system, Prog. Theor. Phys. 62 (1979), 370-378.

[25] V.E. Zakharov, Collapse of Langmuir waves, Sov. Phys. JETP 35 (1972), 908-914.

Yu-Zhu Wang

School of Mathematics and Information Sciences,

North China University of Water Resources and Electric Power

Zhengzhou 450011, China

E-mail: yuzhu108@163.com

Wenxu Ge

School of Mathematics and Information Sciences,

North China University of Water Resources and Electric Power

Zhengzhou 450011, China 